Mathematical Medicine and Biology (2016) 33, 1-28

doi:10.1093/imammb/dqu020

Advance Access publication on October 20, 2014

\title{
Modelling cholesterol effects on the dynamics of the hypothalamic-pituitary-adrenal (HPA) axis
}

\author{
VLADIMIR M. MARKOVIĆ* \\ Faculty of Physical Chemistry, University of Belgrade, Studentski trg 12-16, 11000 Belgrade, Serbia \\ *Corresponding author: Email: vmarkovic@ffh.bg.ac.rs \\ ŽELJKo ČUPIĆ \\ Department of Catalysis and Chemical Engineering, Institute of Chemistry, Technology and \\ Metallurgy, University of Belgrade, Njegoševa 12, 11000 Belgrade, Serbia \\ Stevan Maćešić, Ana Stanojević \\ Faculty of Physical Chemistry, University of Belgrade, Studentski trg 12-16, 11000 Belgrade, Serbia \\ Vladana Vukojević* \\ Department of Clinical Neuroscience, Karolinska Institutet, Center for Molecular Medicine CMM \\ L8:01, 17176 Stockholm, Sweden \\ *Corresponding author: Email: vladana.vukojevic@ki.se
}

AND

\section{LJiLJana KolaR-Anić}

Faculty of Physical Chemistry, University of Belgrade, Studentski trg 12-16, 11000 Belgrade, Serbia and Department of Catalysis and Chemical Engineering, Institute of Chemistry, Technology and Metallurgy, University of Belgrade, Njegoševa 12, 11000 Belgrade, Serbia

[Received on 30 July 2013; revised on 22 July 2014; accepted on 9 September 2014]

A mathematical model of the hypothalamic-pituitary-adrenal (HPA) axis with cholesterol as a dynamical variable was derived to investigate the effects of cholesterol, the primary precursor of all steroid hormones, on the ultradian and circadian HPA axis activity. To develop the model, the parameter space was systematically examined by stoichiometric network analysis to identify conditions for ultradian oscillations, determine conditions under which dynamic transitions, i.e. bifurcations occur and identify bifurcation types. The bifurcations were further characterized using numerical simulations. Model predictions agree well with empirical findings reported in the literature, indicating that cholesterol levels may critically affect the global dynamics of the HPA axis. The proposed model provides a base for better understanding of experimental observations, it may be used as a tool for designing experiments and offers useful insights into the characteristics of basic dynamic regulatory mechanisms that, when impaired, may lead to the development of some modern-lifestyle-associated diseases.

Keywords: hypothalamic-pituitary-adrenal (HPA) axis; cholesterol; circadian and ultradian rhythms; cortisol; dynamical diseases; stoichiometric network analysis. 


\section{Introduction}

The hypothalamic-pituitary-adrenal (HPA) axis is a complex and highly dynamic neuroendocrine system of vital importance for maintaining homeostasis of metabolic functions at the organism level (Miller \& Chrousos, 2001; Lightman et al., 2002; Smith \& Vale, 2006). The HPA axis integrates the function of the hypothalamus, pituitary and adrenal glands through the action of steroid and peptide hormones. The main representative of steroid hormones implicated in the HPA axis in humans is cortisol (CORT), and the most relevant peptide hormones are the corticotropin-releasing hormone (CRH) and the adrenocorticotropic hormone (ACTH) (Miller \& Chrousos, 2001; Lightman et al., 2002). Cortisol, the end product of steroidogenesis and the chief effector steroid hormone in humans exhibits both positive and negative feedback effects on the HPA axis dynamics via its action on glucocorticoid receptors (GR) and mineralocorticoid receptors (MR) (Kellendonk et al., 2002; Makino et al., 2002; Schulkin et al., 1998). Due to the complex interplay between these feedback mechanisms and the coupling with the circadian clock system, the temporal evolution of cortisol concentration in blood plasma is complex, exhibiting ultradian oscillations that are superimposed on the circadian ones. ${ }^{1}$ In humans, the period of cortisol ultradian oscillations can be in the range between 20 and $120 \mathrm{~min}$, while the circadian period is 24 h (Tsigos \& Chrousos, 2002; Hartmann et al., 1997; Gavrila et al., 2003).

It has become well accepted that adequate oscillatory dynamics of cortisol and other HPA axis hormones is necessary for HPA axis function and effective maintenance of homeostasis under normal conditions and stress (Lightman \& Conway-Campbell, 2010). To uphold the oscillatory dynamics, interactions comprising the HPA axis must proceed under conditions far from thermodynamic equilibrium and the concentrations of all HPA axis hormones must be finely tuned. In that regard, derangements in hormone concentrations may significantly alter the oscillatory dynamics of the HPA axis, decreasing the organism's capacity to maintain homeostasis and cope with stress.

Cholesterol, the only source of steroid hormones, is a powerful modulator of steroid hormones concentration, and hence may impinge on the HPA axis dynamics. However, the number of studies that directly investigate the effects of cholesterol on the HPA axis dynamics is not very extensive (Auvinen et al., 2011, 2012; García-Prieto et al., 2007; Lomax et al., 2013). A systematic review by Auvinen et al. (2011) shows the complexity that is associated with such studies and the difficulty to reach unison conclusions due to the intricate nature of the problem and large variations in experimental design.

To understand the interactions between cholesterol and the HPA axis and elucidate the mechanisms through which these interactions are integrated to yield a coherent HPA axis response, integral approaches by mathematical modelling and numerical simulations are needed. The aim of mathematical modelling is to systematically reduce the complexity of the investigated system, i.e. reduce the number of variables to a manageable level, and derive a concise representation of the HPA axis function in the form of a model with good predictive potential. When this goal is achieved, mathematical modelling and numerical simulations along with dynamical systems theory (Izhikevich, 2000; Ingalls, 2013) enable us also to study cholesterol effects on the HPA axis with high precision and predict the temporal evolution of the HPA axis under conditions that are difficult to address experimentally.

To date, several models of the HPA axis have been proposed (Dempsher et al., 1984; Liu et al., 1999; Keenan et al., 2001; Kyrylov et al., 2005; Savić \& Jelić, 2005; Lenbury \& Pornsawad, 2005; Jelić et al., 2005; Gupta et al., 2007; Bairagi et al., 2008; McAuley et al., 2009; Conrad et al., 2009; Walker et al., 2010; Vinther et al., 2011; Marković et al., 2011b; Maćešić et al., 2012; Scheff et al., 2012; Sriram et al., 2012; Postnova et al., 2013; Andersen et al., 2013; Moreno-Ramos et al., 2013;

\footnotetext{
${ }^{1}$ Ultradian rhythms have a period shorter than $24 \mathrm{~h}$, whereas circadian are characterised by periods that are close to $24 \mathrm{~h}$.
} 
Zarzer et al., 2013). The majority of models include cortisol, ACTH and CRH as key dynamic variables to describe the HPA axis dynamics. Depending on the purpose of the study, some models include also other species, such as cortisol bound to cortisol binding globulin and albumin (Liu et al., 1999; Kyrylov et al., 2005), GR (Gupta et al., 2007; Walker et al., 2010; Sriram et al., 2012; Scheff et al., 2012; Zarzer et al., 2013), aldosterone (ALDO) (Jelić et al., 2005; Marković et al., 2011b). However, cholesterol, the only precursor of cortisol and all other steroid hormones has been introduced as a dynamic variable in a few models only. The mechanistic model proposed by Dempsher et al. (1984) contains cholesterol, ACTH, cortisol, enzymes and intermediates involved in cortisol biosynthesis. Yet, this model focuses on adrenal steroidogenesis and ultradian cortisol oscillations have not been considered. The model proposed by Meyer-Hermann et al. (2009) contains cortisol and cholesterol, while CRH and ACTH have not been explicitly regarded as dynamic variables. This model simulates the circadian oscillatory dynamics, while the ultradian oscillations have not been taken into account. Models by Breen et al. $(2010,2011)$ include cholesterol, cortisol, aldosterone and adrenal steroid hormones as dynamic variables, but do not include higher system signals, thus neglecting CRH and ACTH. Due to the absence of feedback mechanisms, these two models do not emulate well neither circadian nor ultradian oscillatory dynamics of cortisol. This lack of succinct models that emulate in detail the cholesterol effect on the HPA axis dynamics at the organism level prompted us to develop a new model that includes cholesterol and other main hormones of the HPA axis, CRH, ACTH, cortisol and aldosterone as dynamic variables, and emulates well their complex oscillatory behaviour, comprising both circadian and ultradian oscillations.

\section{Mathematical Model Development}

\subsection{Mathematical description of the kinetics of biochemical pathways underlying the model of HPA axis activity}

In our previous work (Jelić et al., 2005; Marković et al., 2011b), we have developed a 4D stoichiometric model of HPA axis activity with concentrations of CRH ([CRH]), ACTH ([ACTH]), cortisol ([CORT]) and aldosterone ([ALDO]) as dynamic variables. We have shown that this model exhibits sustained ultradian oscillations of cortisol, ACTH and aldosterone. When coupled to the circadian clock function, this model also emulated the complex superposition of ultradian oscillations on the circadian ones and demonstrated strong predictive capacity and potential for investigating the HPA axis response under various acute and chronic stress conditions (Jelić et al., 2008, 2009; Marković et al., 2011a). Building on this initial model, we introduced concentration of cholesterol ([CHOL]) as the fifth dynamical variable. A diagram showing the relationship between key model variables is presented in Fig. 1(a), while the mathematical model is given in Table 1.

The new model (Table 1) consists of 13 reaction steps, which concisely represent the most important experimentally characterized pathways that epitomize the HPA axis. Reaction steps (R1), (R2) and (R3) describe the biosynthesis of cholesterol, CRH and aldosterone, respectively. Reaction step (R1) exemplifies cholesterol biosynthesis in the liver and the gastrointestinal tract, absorption of dietary cholesterol and receptor-mediated uptake and internalization of plasma lipoprotein-bound cholesterol (DeBoseBoyd, 2008; Lu et al., 2001; Hu et al., 2010). CRH production from the hypothalamic paraventricular nucleus is represented by (R2) and aldosterone production under the renin-angiotensin system control by step (R3). Reaction step (R4) describes the CRH stimulated ACTH production from the pituitary. Reaction steps (R5) and (R6) summarize the multifaceted ACTH-mediated cortisol steroidogenesis in the zona fasciculata (R5) and aldosterone steroidogenesis in the zona glomerulosa (R6) of the 
(a)

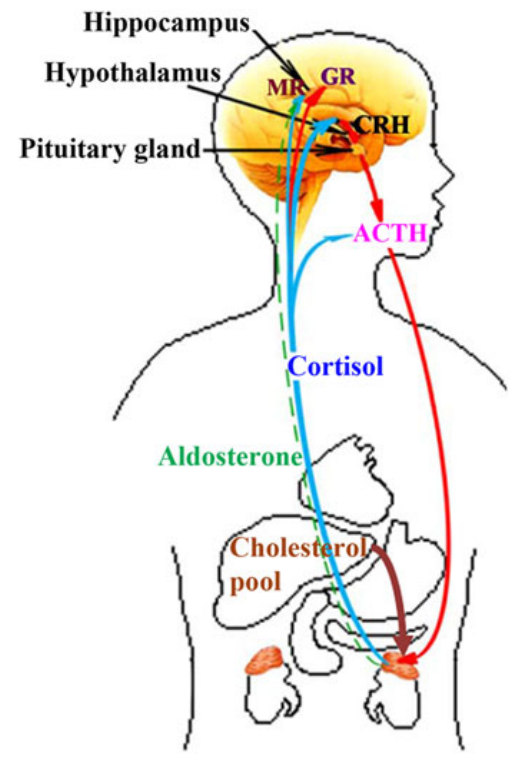

(b)

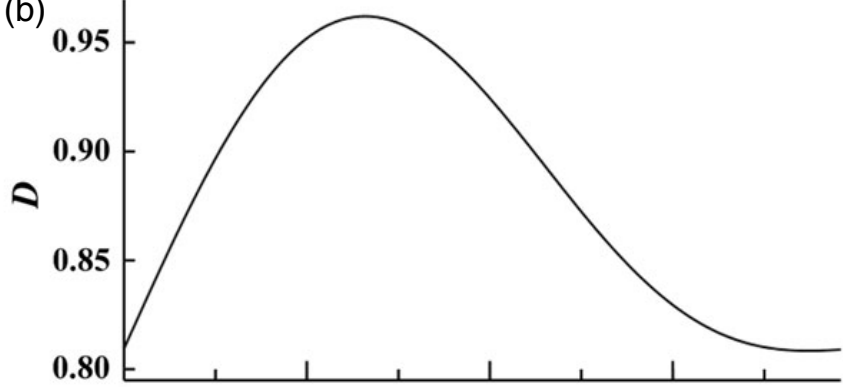

(c)

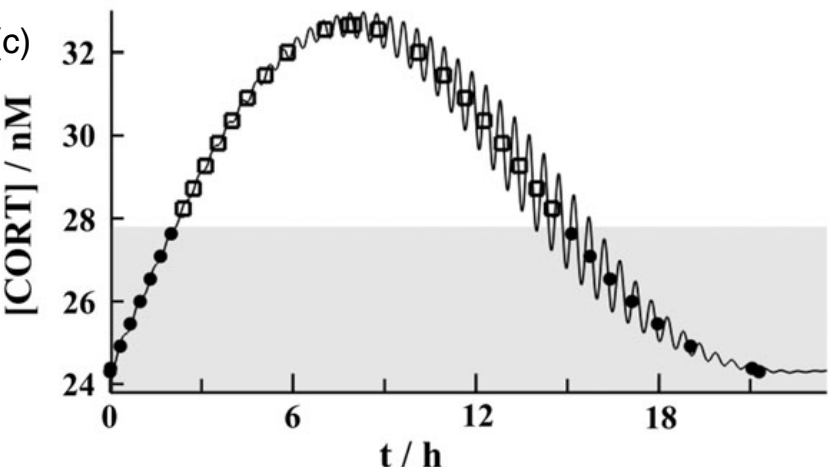

FIG. 1. (a) Schematic presentation of the relationship between key dynamical variables in the model of cholesterol effects on the hypothalamic-pituitary-adrenal (HPA) axis dynamics. The arrows represent interactions between major anatomical components implicated in the HPA axis dynamics, conveyed by the model variables cholesterol, CRH, ACTH, cortisol and aldosterone (details of these interactions, i.e. reaction pathways comprising the model are given in the main text of Section 1). (b) The circadian function $D$ (see Section 2.3). (c) Coupling between circadian and ultradian oscillations introduces a delay in the onset/termination of ultradian oscillations. At the borders of the shaded area, the ultradian stationary state changes its stability by passing through a supercritical Andronov-Hopf bifurcation (dots indicate stable and squares unstable ultradian stationary states of cortisol concentration); the instability condition was derived according to SNA (see Section 2.2). Time is given in hours (h) and molar concentration in $\mathrm{M}=\mathrm{mol} \mathrm{dm}{ }^{-3}$ (these abbreviations were used throughout).

adrenal cortex and their secretion into the global circulation (Miller \& Auchus, 2011; Hu et al., 2010; Gallo-Payet \& Payet, 2003). Reaction step (R7) describes the complex cortisol-mediated positive feedback (feed-forward) regulation of the HPA axis in the form of cubic autocatalysis. Reaction step (R8) describes the cortisol-mediated negative feedback regulation of the HPA axis in the form of quadratic autoinhibition. More detailed explanation on how these complex reactions were derived is given in Jelić et al. (2005). Reaction steps (R9-R13) describe the elimination of cholesterol, CRH, ACTH, cortisol and aldosterone, respectively. Notably, reaction step (R9) entails several processes that reduce the excess of free cholesterol to normal physiological levels, such as cholesterol esterification and storage in lipid droplets, cholesterol incorporation in cellular membranes (membrane biogenesis), production of bile acids, oxysterols, vitamin D and other steroid hormones (Hu et al., 2010; Redinger, 2003). $\mathrm{P}_{n}$ $(n=1, \ldots, 5)$ are elimination products of corresponding species, which have no effect on the HPA axis dynamics.

Temporal dynamics of the concentrations of all species is governed by a system of ordinary differential equations (ODE) derived from reaction steps shown in Table 1 by the law of 
TABLE 1 Model of the HPA axis activity with cholesterol as a dynamical variable

\begin{tabular}{lll}
$\stackrel{k_{1}}{\longrightarrow}$ CHOL & $k_{1}=1.38 \times 10^{-4} \mathrm{~mol} \mathrm{dm}^{-3} \mathrm{~min}^{-1}$ & (R1) \\
$\stackrel{k_{2}}{\longrightarrow}$ CRH & $k_{2}=1.83 \times 10^{-8} \mathrm{~mol} \mathrm{dm}^{-3} \mathrm{~min}^{-1}$ & (R2) \\
$\stackrel{k_{3}}{\longrightarrow}$ ALDO & $k_{3}=6.09 \times 10^{-11} \mathrm{~mol} \mathrm{dm}^{-3} \mathrm{~min}^{-1}$ & (R3) \\
$\mathrm{CRH} \stackrel{k_{4}}{\longrightarrow}$ ACTH & $k_{4}=1.83 \times 10^{4} \mathrm{~min}^{-1}$ & (R4) \\
$\mathrm{CHOL}+\mathrm{ACTH} \stackrel{k_{5}}{\longrightarrow}$ CORT & $k_{5}=11.94 \mathrm{~mol}^{-1} \mathrm{dm}^{3} \mathrm{~min}^{-1}$ & (R5) \\
$\mathrm{CHOL}+\mathrm{ACTH} \stackrel{k_{6}}{\longrightarrow}$ ALDO & $k_{6}=9.552 \times 10^{-2} \mathrm{~mol}^{-1} \mathrm{dm}^{3} \mathrm{~min}^{-1}$ & (R6) \\
$\mathrm{ACTH}+2 \mathrm{CORT} \stackrel{k_{7}}{\longrightarrow}$ 3CORT & $k_{7}=1.26 \times 10^{14} \mathrm{~mol}^{-2} \mathrm{dm}^{6} \mathrm{~min}^{-1}$ & (R7) \\
$\mathrm{ALDO}+2 \mathrm{CORT} \stackrel{k_{8}}{\longrightarrow}$ CORT & $k_{8}=7.05 \times 10^{12} \mathrm{~mol}^{-2} \mathrm{dm}^{6} \mathrm{~min}^{-1}$ & (R8) \\
$\mathrm{CHOL} \stackrel{k_{9}}{\longrightarrow} \mathrm{P}_{1}$ & $k_{9}=4.5 \times 10^{-2} \mathrm{~min}^{-1}$ & (R9) \\
$\mathrm{CRH} \stackrel{k_{10}}{\longrightarrow} \mathrm{P}_{2}$ & $k_{10}=1.1 \times 10^{-1} \mathrm{~min}^{-1}$ & (R10) \\
$\mathrm{ACTH} \stackrel{k_{11}}{\longrightarrow} \mathrm{P}_{3}$ & $k_{11}=5.35 \times 10^{-2} \mathrm{~min}^{-1}$ & (R11) \\
$\mathrm{CORT} \stackrel{k_{12}}{\longrightarrow} \mathrm{P}_{4}$ & $k_{12}=4.1 \times 10^{-1} \mathrm{~min}^{-1}$ & (R12) \\
$\mathrm{ALDO} \stackrel{k_{13}}{\longrightarrow} \mathrm{P}_{5}$ & $k_{13}=1.35 \times 10^{-1} \mathrm{~min}^{-1}$ & (R13) \\
\hline
\end{tabular}

mass action:

$$
\begin{aligned}
& \frac{\mathrm{d}[\mathrm{CHOL}]}{\mathrm{d} t}=k_{1}-\left(k_{5}+k_{6}\right)[\mathrm{CHOL}][\mathrm{ACTH}]-k_{9}[\mathrm{CHOL}] \\
& \frac{\mathrm{d}[\mathrm{CRH}]}{\mathrm{d} t}=k_{2}-\left(k_{4}+k_{10}\right)[\mathrm{CRH}] \\
& \frac{\mathrm{d}[\mathrm{ACTH}]}{\mathrm{d} t}=k_{4}[\mathrm{CRH}]-\left(k_{5}+k_{6}\right)[\mathrm{CHOL}][\mathrm{ACTH}]-k_{7}[\mathrm{ACTH}][\mathrm{CORT}]^{2}-k_{11}[\mathrm{ACTH}] \\
& \frac{\mathrm{d}[\mathrm{CORT}]}{\mathrm{d} t}=k_{5}[\mathrm{CHOL}][\mathrm{ACTH}]+k_{7}[\mathrm{ACTH}][\mathrm{CORT}]^{2}-k_{8}[\mathrm{ALDO}][\mathrm{CORT}]^{2}-k_{12}[\mathrm{CORT}] \\
& \frac{\mathrm{d}[\mathrm{ALDO}]}{\mathrm{d} t}=k_{3}+k_{6}[\mathrm{CHOL}][\mathrm{ACTH}]-k_{8}[\mathrm{ALDO}][\mathrm{CORT}]^{2}-k_{13}[\mathrm{ALDO}] .
\end{aligned}
$$

In (1a-1e), the concentrations of cholesterol, CRH, ACTH, cortisol and aldosterone are denoted by $[\mathrm{CHOL}],[\mathrm{CRH}],[\mathrm{ACTH}],[\mathrm{CORT}]$ and $[\mathrm{ALDO}]$, respectively, and the related kinetic rate constants are designated by $k_{m}, m=1-13$.

2.2 Determination of oscillatory regions by systematic analysis of the parameter space using stoichiometric network analysis

To determine the oscillatory region, the parameter space was systematically examined by stoichiometric network analysis (SNA), a general flux balance analysis method that relies on the analysis of stoichiometric characteristics of a network (Clarke, 1980, 1988). A comprehensive derivation of general equations and details of SNA application for the analysis of bifurcation mechanisms can be found elsewhere (Clarke, 1980, 1988; Schmitz et al., 2008; Kolar-Anić et al., 2010; Čupić et al., 2011); we present here the most important steps that relate to the analysis of the model. 
The first step in SNA is to detect steady-state reaction pathways in the model (R1)-(R13) that are inherently coupled through stoichiometric constraints. This derivation, presented in Appendix, yields the following relationships:

$$
\begin{aligned}
v_{1, \mathrm{ss}} & =v_{5, \mathrm{ss}}+v_{6, \mathrm{ss}}+v_{9, \mathrm{ss}} \\
v_{2, \mathrm{ss}} & =v_{4, \mathrm{ss}}+v_{10, \mathrm{ss}} \\
v_{3, \mathrm{ss}}+v_{6, \mathrm{ss}} & =v_{8, \mathrm{ss}}+v_{13, \mathrm{ss}} \\
v_{5, \mathrm{ss}}+v_{7, \mathrm{ss}} & =v_{8, \mathrm{ss}}+v_{12, \mathrm{ss}} \\
v_{4, \mathrm{ss}} & =v_{5, \mathrm{ss}}+v_{6, \mathrm{ss}}+v_{7, \mathrm{ss}}+v_{11, \mathrm{ss}},
\end{aligned}
$$

where $v_{\mathrm{m}, \mathrm{ss}}$ is the reaction rate of reaction $m(m=1-13)$ at the steady state.

The next step in SNA is to investigate the stability of the network's motion around a steady state after an infinitely small perturbation. To this end, one uses the linearized dynamics operator near the steady state (the Jacobian) $\mathbf{J}$ :

$$
\frac{\mathrm{d} x}{\mathrm{~d} t}=\mathbf{J} \boldsymbol{x}
$$

where $\mathbf{x}$ is the concentration vector whose components $\left[\mathrm{X}_{n}\right]$ are concentrations of intermediary chemical species of a reaction network (here, $n=1,2, \ldots, 5$, and $\left[\mathrm{X}_{1}\right]=[\mathrm{CHOL}],\left[\mathrm{X}_{2}\right]=[\mathrm{CRH}],\left[\mathrm{X}_{3}\right]=$ $[\mathrm{ACTH}],\left[\mathrm{X}_{4}\right]=[\mathrm{CORT}],\left[\mathrm{X}_{5}\right]=[\mathrm{ALDO}]$; see Appendix for details).

The matrix operator $\mathbf{J}$ is defined in SNA (Clarke, 1980, 1988; Čupić et al., 2011) by:

$$
\mathbf{J}=-\mathbf{V}(\boldsymbol{j}) \operatorname{diag} \boldsymbol{h},
$$

where $\mathbf{h}$ is a vector of reciprocal steady-state concentrations, whose components $\mathrm{h}_{i}$ are reciprocal steadystate concentrations of the intermediates, $\operatorname{diag} \boldsymbol{h}$ is its diagonal matrix, while $\mathbf{V}(\boldsymbol{j})$ is a current rate matrix, given by the expression:

$$
\mathbf{V}(\boldsymbol{j})=-S \operatorname{diag}(\mathbf{E} \mathbf{j}) \mathbf{K}^{\mathrm{T}},
$$

where $\mathbf{S}$ is the stoichiometric matrix, i.e. an operator whose elements are stoichiometric coefficients, $\mathrm{S}_{n, m}$, defined as $\mathrm{S}_{n, m}=\mathrm{s}_{n, m}^{\mathrm{D}}-\mathrm{s}_{n, m}^{\mathrm{L}}$, where, $\mathrm{s}_{n, m}^{\mathrm{L}}$ and $\mathrm{s}_{n, m}^{\mathrm{D}}$, represent stoichiometric coefficients of species $\mathrm{X}_{n}$ on the left (L) and right (D) sides of the chemical equation for Rm, respectively, where $n$ stands for total number of intermediary species and $m$ is total number of reactions in the reaction network (see Appendix for details). If the underlying kinetics occurs according to the law of mass action, $\mathbf{K}^{\mathrm{T}}$ in (5) represents the transpose of the matrix of reaction orders, whose elements are general stoichiometric coefficients of species $\mathrm{X}_{n}$ standing on the left side of the reaction $\mathrm{R} m, s_{n, m}^{\mathrm{L}}$.

Here, elements of diag $\boldsymbol{h}, \boldsymbol{h}=\left[h_{1}, h_{2}, \ldots h_{5}\right]$, are the reciprocals of steady-state concentrations of the model's intermediary chemical species cholesterol, CRH, ACTH, cortisol and aldosterone, respectively, whereas the matrix $\mathbf{K}$ is:

$$
\begin{array}{ccccccccccccc}
\mathrm{R} 1 & \mathrm{R} 2 & \mathrm{R} 3 & \mathrm{R} 4 & \mathrm{R} 5 & \mathrm{R} 6 & \mathrm{R} 7 & \mathrm{R} 8 & \mathrm{R} 9 & \mathrm{R} 10 & \mathrm{R} 11 \mathrm{R} 12 & \mathrm{R} 13 \\
\mathbf{K} & {\left[\begin{array}{ccccccccccccc}
0 & 0 & 0 & 0 & 1 & 1 & 0 & 0 & 1 & 0 & 0 & 0 & 0 \\
0 & 0 & 0 & 1 & 0 & 0 & 0 & 0 & 0 & 1 & 0 & 0 & 0 \\
0 & 0 & 0 & 0 & 1 & 1 & 1 & 0 & 0 & 0 & 1 & 0 & 0 \\
0 & 0 & 0 & 0 & 0 & 0 & 2 & 2 & 0 & 0 & 0 & 1 & 0 \\
0 & 0 & 0 & 0 & 0 & 0 & 0 & 1 & 0 & 0 & 0 & 0 & 1
\end{array}\right][\text { [CRH] }} \\
{[\mathrm{CHCTH}]} \\
{[\mathrm{CORT}]}
\end{array}
$$


By substituting matrices K (6), S (Appendix, A.3) and $\mathbf{E}$ (Appendix, A.5) in (5), current rate matrix $\mathbf{V}(\boldsymbol{j})$ can be derived. The elements of the matrix $\mathbf{V}(\boldsymbol{j})$ are

$$
\begin{aligned}
& V(1,1)=j_{1}+j_{2}+j_{5}+2 j_{9}+j_{10}+j_{11} \\
& V(1,3)=j_{1}+j_{5}+2 j_{9}+j_{10}+j_{11} \\
& V(2,2)=j_{1}+j_{3}+j_{4}+j_{5}+j_{7}+j_{8}+2 j_{9}+j_{10}+2 j_{11} \\
& V(3,1)=j_{1}+j_{5}+2 j_{9}+j_{10}+j_{11} \\
& V(3,2)=-j_{1}-j_{4}-j_{5}-j_{7}-j_{8}-2 j_{9}-j_{10}-2 j_{11} \\
& V(3,3)=j_{1}+j_{4}+j_{5}+j_{7}+j_{8}+2 j_{9}+j_{10}+2 j_{11} \\
& V(3,4)=2 j_{7}+2 j_{8}+2 j_{11} \\
& V(4,1)=-j_{1}-j_{5}-j_{9} \\
& V(4,3)=-j_{1}-j_{5}-j_{7}-j_{8}-j_{9}-j_{11} \\
& V(4,4)=2 j_{1}+j_{5}-j_{8}+2 j_{9} \\
& V(4,5)=j_{1}+j_{7}+j_{9}+j_{11} \\
& V(5,1)=-j_{9}-j_{10}-j_{11} \\
& V(5,3)=-j_{9}-j_{10}-j_{11} \\
& V(5,4)=2 j_{1}+2 j_{7}+2 j_{9}+2 j_{11} \\
& V(5,5)=j_{1}+j_{6}+j_{7}+j_{9}+j_{10}+j_{11} \\
& V(1,2)=V(1,4)=V(1,5)=V(2,1)=V(2,3)=V(2,4) \\
& =V(2,5)=V(3,5)=V(4,2)=V(5,2)=0
\end{aligned}
$$

Since the stability of the stationary state is defined by the eigenvalues $\lambda$ of the linearized operator $\mathbf{J}$, which are given as roots of the characteristic polynomial, for the model analysed here with five independent intermediate species i.e. five dynamic variables, the characteristic polynomial of the fifth order has to be solved:

$$
\operatorname{Det}[\lambda \mathbf{I}-\mathbf{J}]=\lambda^{5}+\alpha_{1} \lambda^{4}+\lambda^{3} \alpha_{2}+\lambda^{2} \alpha_{3}+\lambda \alpha_{4}+\alpha_{5}
$$

where $\alpha_{i}(i=1,2, \ldots, 5)$ is the coefficient of the corresponding eigenvalue $\lambda^{5-i}$, and $\mathbf{I}$ is the unit matrix. By convention, $\alpha_{0}=1$.

Coefficients $\alpha_{i}$ of the characteristic equation (8) represent the sum of all diagonal minors $\mathrm{M}$ of dimensions $i \times i(i=1, \ldots, 5)$ of the matrix $\mathbf{V}(j)$ multiplied by corresponding sets of reciprocal concentrations $h_{i}$ (Clarke, 1980, 1988; Čupić et al., 2011). For instance, coefficient $\alpha_{3}$ is the sum of all combinations of diagonal minors of dimension $3 \times 3$, i.e. minors of the third order multiplied by its corresponding product of reciprocal concentrations:

$$
\begin{aligned}
\alpha_{3}= & M_{123} h_{1} h_{2} h_{3}+M_{124} h_{1} h_{2} h_{4}+M_{125} h_{1} h_{2} h_{5}+M_{134} h_{1} h_{3} h_{4}+M_{135} h_{1} h_{3} h_{5}+M_{145} h_{1} h_{4} h_{5} \\
& +M_{234} h_{2} h_{3} h_{4}+M_{235} h_{2} h_{3} h_{5}+M_{245} h_{2} h_{4} h_{5}+M_{345} h_{3} h_{4} h_{5} .
\end{aligned}
$$


Although the derivation of the characteristic equation is precisely defined, the obtained fifth order equation (8) is generally not possible to solve analytically, requiring numerical analysis of particular cases with defined parameter values. To circumvent this obstacle, SNA often concludes after calculating the coefficients $\alpha_{i}$, when the so-called alpha approximation is employed. In many instances, SNA concludes even earlier, after finding diagonal minors $\mathbf{M}$ of the matrix $\mathbf{V}(j)$ that contain negative terms. For many applications, such approximations can yield instability conditions that are sufficiently accurate (Schmitz et al., 2008; Kolar-Anić et al., 2010; Čupić et al., 2011; Marković et al., 2011b; Maćešić et al., 2012). Otherwise, exact instability conditions are acquired based on the Routh-Hurwitz criterion. According to this criterion the number of eigenvalues with positive real parts is equal to the number of sign changes in the Routh array (Clarke \& Jiang, 1993):

$$
R=\left(1, \Delta_{1}, \frac{\Delta_{2}}{\Delta_{1}}, \frac{\Delta_{3}}{\Delta_{2}}, \ldots, \frac{\Delta_{n}}{\Delta_{n-1}}\right)
$$

where $\Delta_{i}, i=1, \ldots, n$, is $i$ th Hurwitz determinant, defined as the determinant of the leading principal minor of the Hurwitz matrix $\mathbf{H}$.

For the analysed model, where $n=5$, the Hurwitz matrix is

$$
\mathbf{H}=\left[\begin{array}{ccccc}
\alpha_{1} & \alpha_{3} & \alpha_{5} & 0 & 0 \\
1 & \alpha_{2} & \alpha_{4} & 0 & 0 \\
0 & \alpha_{1} & \alpha_{3} & \alpha_{5} & 0 \\
0 & 1 & \alpha_{2} & \alpha_{4} & 0 \\
0 & 0 & \alpha_{1} & \alpha_{3} & \alpha_{5}
\end{array}\right]
$$

Obviously, $\alpha_{i}=0$, for $i>5$, whereas coefficients $\alpha_{i}, i \leqslant 5$, of the Hurwitz matrix $\mathbf{H}$ can be written differently, depending on the applied method of analysis. Straightforward linearization of the system of ODE (1a-1e) would yield expressions that are functions of the reaction rates and steady-state concentrations. By SNA, the same coefficients would be obtained as functions of current rates and reciprocal concentrations in a steady state. In either way, a fifth order characteristic equation would be obtained that needs to be solved numerically. There are no principal differences between these approaches, but the advantage of using SNA instead of direct linearization of equation lies in the ease with which initial conditions for numerical solutions can be found. In a large parameter space, which in the case of the proposed model has 13 reactions and hence 13 corresponding rate constants, finding convenient initial conditions for numerical analysis is not easy. Using SNA, the search for initial conditions is facilitated by the so-called alpha approximation, which provides a 'frame' for looking for the instability, making it possible to identify an area in the parameter space where unstable steady states could be numerically found and characterized by evaluating the eigenvalues $\lambda$ from (8). Direct analysis of (1a-1e) does not yield this advantage.

Let us underline here that it is not necessary to calculate precisely the eigenvalues of the linearized operator to determine the stability of a steady state. Rather, the steady state is stable if all Hurwitz determinants are positive.

The Hurwitz matrix gives also conditions for the appearance of the Andronov-Hopf bifurcation, which is of great importance because it is a commonly encountered source of oscillations in dynamical system. The Andronov-Hopf bifurcation is a local bifurcation through which a dynamical system loses/gains stability as a pair of complex conjugate eigenvalues crosses the imaginary axis. This bifurcation indicates appearance/disappearance of periodic behaviour. The Andronov-Hopf bifurcation occurs 
when $\Delta_{n-1}=0$ (Clarke \& Jiang, 1993; Liu, 1994), which for the analysed model is when:

$$
\Delta_{4}=\left[\begin{array}{cccc}
\alpha_{1} & \alpha_{3} & \alpha_{5} & 0 \\
1 & \alpha_{2} & \alpha_{4} & 0 \\
0 & \alpha_{1} & \alpha_{3} & \alpha_{5} \\
0 & 1 & \alpha_{2} & \alpha_{4}
\end{array}\right]=0
$$

Moreover, the saddle-node bifurcation can be identified from $\alpha_{n}=0$, as shown by Clarke $\&$ Jiang (1993) and Liu (1994), which for the analysed model gives:

$$
\alpha_{5}=0
$$

Beside general problems related with the determination of steady-state stability which were mentioned above, the original SNA, as described by Clarke $(1980,1988)$, gives the coefficients $\alpha_{i}$, derived from the matrix $\mathbf{V}(j)$ ( 7) and subsequent Hurwitz matrices, as functions of the current rates $j_{i}$. As mentioned earlier (Kolar-Anić et al., 2010; Čupić et al., 2011; Marković et al., 2011b; Maćešić et al., 2012), diagonal minors, and, hence, coefficients $\alpha_{i}$, can be completely expressed as functions of reaction rates at the steady states, $v_{m, s s}$. Since reaction rates are experimentally available quantities, the latter form of expressing minors or coefficients $\alpha_{i}$ is not only more convenient to deal with, but also easier to understand and relate to real properties of the system.

As the instability condition depends on reaction rates at the steady state, $v_{m}$,ss, which are functions of rate constants and steady-state concentrations of the reactive species (Appendix, (A.6a-A.6m)), to determine whether it is fulfilled, the steady-state concentrations of the model's intermediary chemical species, cholesterol $\left([\mathrm{CHOL}]_{\mathrm{ss}}\right), \mathrm{CRH}\left([\mathrm{CRH}]_{\mathrm{ss}}\right), \mathrm{ACTH}\left([\mathrm{ACTH}]_{\mathrm{ss}}\right)$, cortisol $\left([\mathrm{CORT}]_{\mathrm{ss}}\right)$ and aldosterone $\left([\mathrm{ALDO}]_{\mathrm{ss}}\right)$, need to be calculated.

The steady-state concentration of cholesterol, obtained by equating (1a) with zero yields:

$$
[\mathrm{CHOL}]_{\mathrm{ss}}=\frac{k_{1}}{[\mathrm{ACTH}]_{\mathrm{ss}}\left(k_{1}+k_{8}\right)+k_{9}},
$$

while, $\mathrm{CRH}$ is obtained by equating (1b) with zero:

$$
[\mathrm{CRH}]_{\mathrm{ss}}=\frac{k_{2}}{k_{4}+k_{10}} .
$$

By equating (1c) with zero and substituting in it (15), the steady-state concentration of ACTH is obtained:

$$
[\mathrm{ACTH}]_{\mathrm{ss}}=\frac{k_{2} k_{4}}{\left(k_{4}+k_{10}\right)\left[\left(k_{5}+k_{6}\right)[\mathrm{CHOL}]_{\mathrm{ss}}+k_{7}\left([\mathrm{CORT}]_{\mathrm{ss}}\right)^{2}+k_{11}\right]},
$$

whereas the steady-state concentration of aldosterone is obtained by equating (1e) with zero:

$$
[\mathrm{ALDO}]_{\mathrm{ss}}=\frac{k_{3}+k_{6}[\mathrm{CHOL}]_{\mathrm{ss}}[\mathrm{ACTH}]_{\mathrm{ss}}}{k_{8}\left([\mathrm{CORT}]_{\mathrm{ss}}\right)^{2}+k_{13}} .
$$


By substituting (14), (16) and (17) in (1d) equalled to zero, an implicit equation for the steady-state cortisol concentration, $x_{4, \mathrm{ss}}$, is obtained:

$$
\begin{aligned}
& {\left[k_{7} k_{8}\left(k_{1}-k_{9}[\mathrm{CHOL}]_{\mathrm{ss}}\right)\right]\left([\mathrm{CORT}]_{\mathrm{ss}}\right)^{4}-\left\{\left[\left(k_{5}+k_{6}\right) k_{8} k_{12}\right][\mathrm{CHOL}]_{\mathrm{ss}}\right\}\left([\mathrm{CORT}]_{\mathrm{ss}}\right)^{3}} \\
& \quad+\left\{\left(k_{6}-k_{5}\right) k_{8} k_{9}\left([\mathrm{CHOL}]_{\mathrm{ss}}\right)^{2}-\left[k_{7} k_{9} k_{13}+k_{3}\left(k_{5}+k_{6}\right) k_{8}-k_{1} k_{8}\left(k_{6}-k_{5}\right)\right][\mathrm{CHOL}]_{\mathrm{ss}}\right\}\left([\mathrm{CORT}]_{\mathrm{ss}}\right)^{2} \\
& \quad-\left\{\left[\left(k_{5}+k_{6}\right) k_{12} k_{13}\right][\mathrm{CHOL}]_{\mathrm{ss}}\right\}[\mathrm{CORT}]_{\mathrm{ss}}-\left(k_{5} k_{9} k_{13}\left([\mathrm{CHOL}]_{\mathrm{ss}}\right)^{2}-k_{1} k_{5} k_{13}[\mathrm{CHOL}]_{\mathrm{ss}}\right)=0 .
\end{aligned}
$$

Equations (14-18) demonstrate that steady-state concentrations of all species in the extended HPA axis model with cholesterol are functions of kinetic rate constants, which means that bifurcation conditions in (12) and (13) can be used to generate instability conditions for the steady states of the focus (19) or saddle type (20), respectively:

$$
\begin{aligned}
& \Delta_{4}<0, \\
& \alpha_{5}<0 .
\end{aligned}
$$

Since inequalities (19) and (20) depend solely on the kinetic rate constants, knowing their values is sufficient condition to determine whether the investigated model of cholesterol effect on the HPA axis activity can have unstable states, which makes this method very convenient for numerical evaluation of steady-states stability. Solutions of the system of ODE (1a-1e) obtained by numerical integration give the concentrations of the dynamical species CHOL, CRH, ACTH, CORT and ALDO and their temporal evolution (Fig. 2).

To determine the oscillatory region and examine the effect of cholesterol concentrations on the ultradian HPA dynamics, rate constants $k_{1}$ and $k_{9}$, related to cholesterol inflow and outflow, respectively, were broadly varied, the steady states were identified using (14-18) and their stability was determined from the instability conditions (19) and (20). The obtained oscillatory domain as a function of $k_{1}$ and $k_{9}$ is presented in Fig. 3.

The upper limit of the instability region in Fig.3 represents Andronov-Hopf bifurcation points for different pairs of $k_{1}$ and $k_{9}$, where the condition given in (19) is satisfied for the only steady state existing in this part of the parameter space. The lower limit represents saddle-loop bifurcations, ${ }^{2}$ where a pair of new steady states appears as a solution of (14-18), one of them being stable (with all Hurwitz matrices being positive), and the other one with saddle type instability ( $\alpha_{5}$ being negative, see (20)).

The rather narrow instability region presented in Fig.3 compellingly shows that oscillatory dynamic states cannot be easily found by guessing, further stressing the importance of methods for systematic global examination of the parameter space, such as SNA.

\subsection{Mathematical description of the coupling between circadian and ultradian oscillations}

The model (R1-R13), in the form presented in Table 1, reflects the ultradian dynamics of the HPA axis (Fig. 2). To account for CRH production, which is governed by the circadian clock system situated in the suprachiasmatic nucleus in the anterior hypothalamus, an extrinsic periodic function $(D)$ has been introduced. Function $D$ is designed to appropriately mimic the asymmetry of the 24 -h rhythm in humans,

\footnotetext{
${ }^{2}$ A saddle-loop or homoclinic bifurcation is an infinite-period bifurcation that occurs when a periodic orbit, i.e. a limit cycle moves closer and closer to a saddle point. At the bifurcation, the cycle touches the saddle point and becomes a homoclinic orbit (Strogatz, 1994).
} 

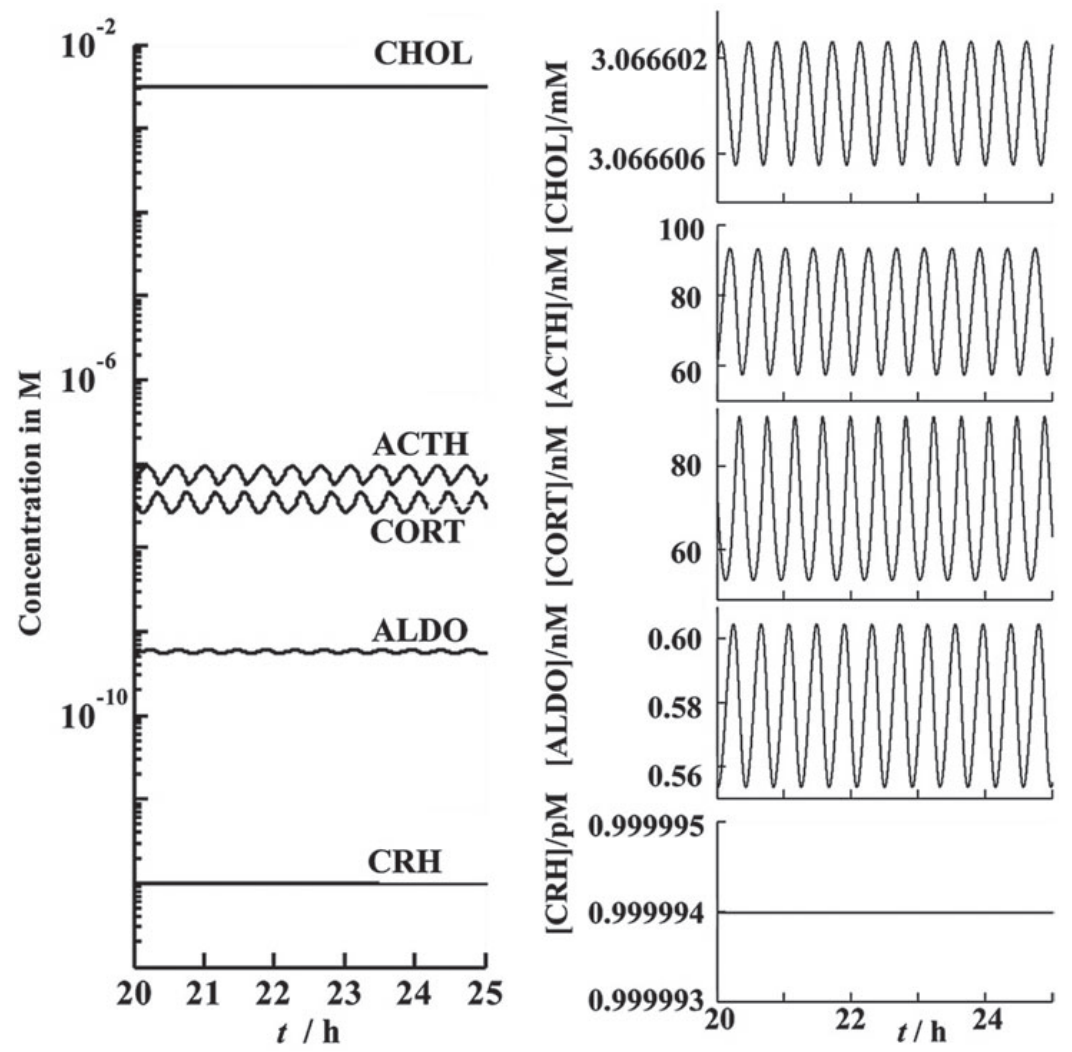

FIG. 2. Time-series showing the temporal dynamics of concentrations of: cholesterol ([CHOL]), CRH ([CRH]), ACTH ([ACTH]), cortisol ([CORT]) and aldosterone ([ALDO]), obtained by numerical integration of ODE (1a-1e) for the values of kinetic rate constants given in Table 1.

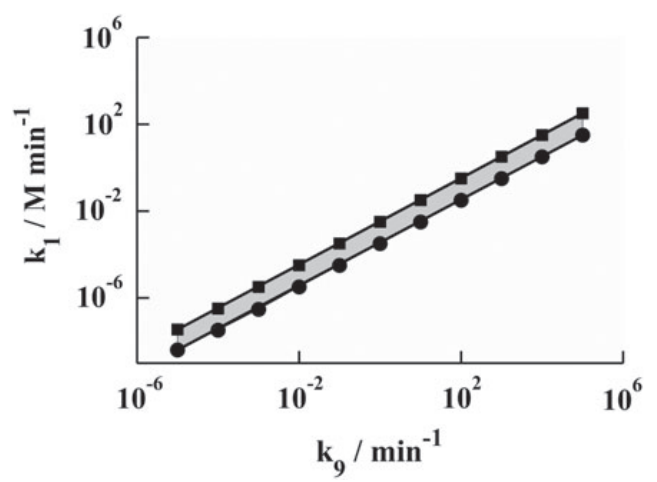

FIG. 3. The oscillatory domain with respect to control parameters $k_{1}$ and $k_{9}$ is confined between the two straight lines. 
with the nocturnal phase lasting 8 h (Fig. 1(b); Marković et al., 2011a):

$$
D=d_{1}-0.079145093 \times d_{2}+\{0.064 \times \sin (2 \pi t / 1440)+0.12 \times \operatorname{abs}[\sin (\pi t / 1440)]\} \times d_{2}
$$

In (21), $t$ is time in minutes and 1440 in the denominator of the trigonometric function argument represents the number of minutes in 1 day, i.e. the period of the circadian oscillation.

The circadian rhythm function $D$ (Fig. 1(b)) is coupled to the model in Table 1 through the CRH production step (R2), transforming the kinetic rate constant $k_{2}$ into a periodic function $k_{2, D}(t)=k_{2} \times D$. Coupling between ultradian and circadian rhythms gives rise to complex daily variations in the concentration of HPA axis hormones, as shown in Fig. 1(c), but does not qualitatively alter the dynamical properties of the model. Instead, coupling between circadian and ultradian rhythms introduces a delay in the onset/termination of ultradian oscillations, giving damped oscillatory evolution even when the steady states are stable. Stable foci (dots) are observed in the shaded region, while unstable foci (squares) are present above this region, for higher cortisol concentrations Fig. 1(c). On the borders of the shaded area the ultradian stationary state changes its stability by passing through a supercritical AndronovHopf bifurcation determined by SNA using (12).

Solutions of the system of ODEs (1a-1e) with $k_{2}$ or $k_{2, D}$ yield for the same set of parameters, that is the same rate constants $\mathrm{k}_{i}, i=1,3-13$ (Table 1) and the same initial conditions, different values of CHOL, CRH, ACTH, CORT and ALDO concentrations, as evident from the results shown in Figs 2 and 4 , respectively.

\section{Results and Discussion}

\subsection{Numerical simulation of cholesterol and HPA axis dynamics}

Serum cholesterol levels vary between individuals, but also in the same individual, where diurnal and ultradian variations have been observed (Jones \& Schoeller, 1990; Peterson et al., 1960; Cirelli et al., 2004). In particular, a pronounced depression of cholesterol synthesis was observed during wakefulness (Jones \& Schoeller, 1990), modelled here as the daytime period, whereas expression of several genes related to the synthesis of cholesterol peaks during the resting phase (Cirelli et al., 2004), modelled here as the nighttime. These experimental findings are correctly accounted for by the model, as evident from the temporal evolution of cholesterol and the HPA axis hormones shown in Fig. 4.

Mean daily levels of cholesterol and selected HPA axis hormones predicted by numerical simulations are compared with their corresponding values predicted by the basic model of Jelic et al. (2005) and basal levels in human plasma (Table 2).

As can be seen from the data presented in Fig. 4 and Table 2, the model emulates diurnal and ultradian variations in cholesterol and HPA axis hormones level, and the concentrations of cholesterol, CRH, cortisol and aldosterone predicted by modelling fall in physiologically relevant ranges. The cholesterol concentration is several orders of magnitude higher than the concentration of CORT and ALDO, which are derived from cholesterol. The amplitude of ultradian cholesterol oscillation is therefore very small in comparison with its mean concentration, whereas the amplitude of CORT and ALDO oscillations may be comparable with their mean daily values. The current model is in better agreement with real measurements than the model without cholesterol previously proposed by Jelić et al. (2005), where both, CRH and ACTH concentrations are several orders of magnitude higher than their actual physiological values. The only discrepancy from real measurements that presently remains unsolved is the concentration of ACTH, which is three orders of magnitude higher. This discrepancy arises due to the conciseness of the model, which does not include peptide precursors of ACTH and other biologically 
(a)

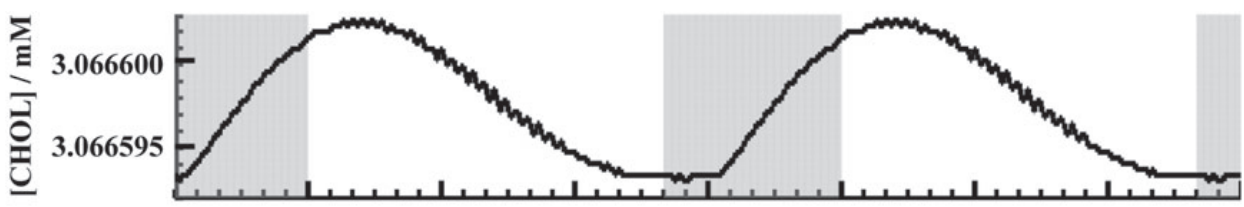

(b)

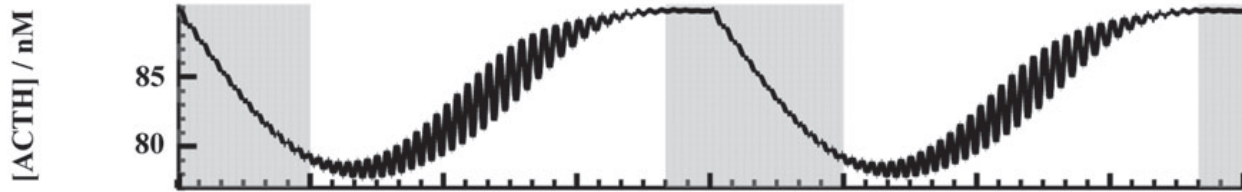

(c)

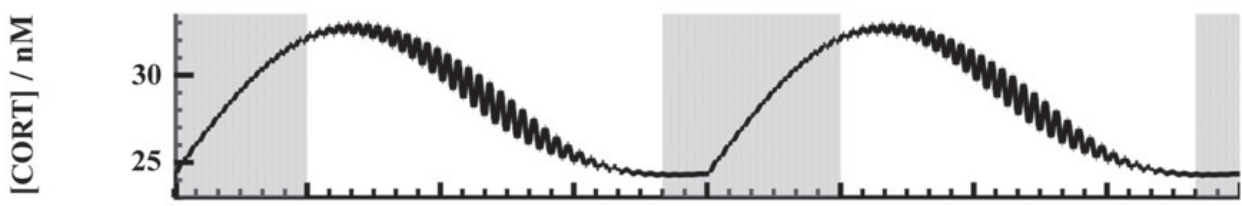

(d)

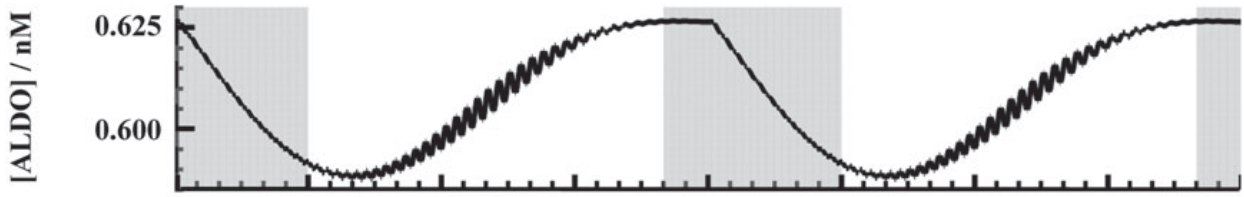

(e)

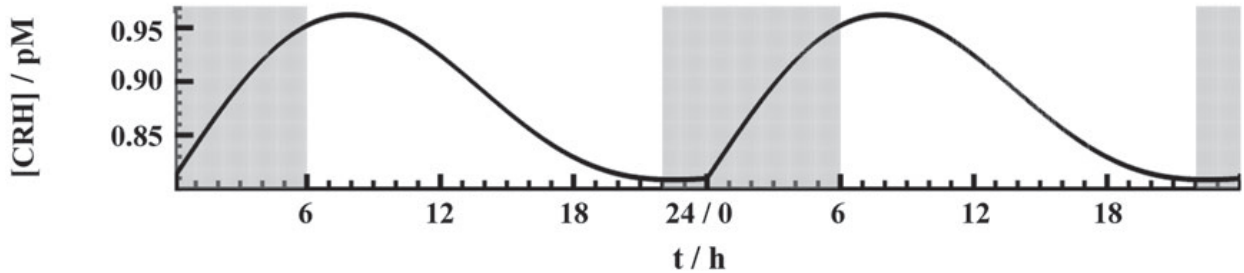

FIG. 4. Numerical simulations of cholesterol and HPA axis dynamics under normal physiological conditions. Temporal evolution of: (a) cholesterol, (b) ACTH, (c) cortisol, (d) aldosterone and (e) CRH showing both diurnal and ultradian rhythmicity. Initial conditions for numerical integration are given in Section 5. The indicated temporal scale reflects concentration variations within 1 day $(24 \mathrm{~h})$. (The actual integration time is a sequence of continuously increasing values, which can be obtained from the values shown in the image by adding for each subsequent day an integer multiple of 24 to the values given for the first day.) The shaded area indicates the rest period, lasting from 22:00 o'clock in the evening to 6:00 o'clock in the morning.

active steroid hormones. Further model enlargement may correct this difference in a similar way as the introduction of cholesterol enabled the adjustment of CRH and ALDO concentrations, which were too high in previous models (Table 2; Jelić et al., 2005, 2008, 2009; Marković et al., 2011a,b).

\subsection{Numerical simulation of HPA axis dynamics under cholesterol regulation}

To assess the potential of the proposed model to emulate the effect of cholesterol on the HPA axis dynamics, kinetic rate constants $k_{1}$, describing cholesterol inflow (due to biosynthesis and resorption from the digestive tract) and $k_{9}$, describing its outflow (due to chemical transformations and elimination), were systematically varied from low to high values in the parameter space domain identified by SNA examination of steady-state stability (Fig. 3, and the last two paragraphs in Section 
TABLE 2 Mean daily levels of cholesterol and HPA axis hormones CRH, ACTH, cortisol and aldosterone predicted by the HPA axis model with cholesterol (Table 1), Jelic et al. (2005) model and in vivo measured normal basal levels. Values refer to normal physiological conditions. $M=\mathrm{mol} \mathrm{dm}^{-3}$

\begin{tabular}{llll}
\hline $\begin{array}{l}\text { Mean } \\
\text { concentration/M }\end{array}$ & $\begin{array}{l}\text { HPA model by } \\
\text { Jelić } \text { et al. }(2005)\end{array}$ & $\begin{array}{l}\text { HPA model with } \\
\text { CHOL }(\text { Table } 1)\end{array}$ & $\begin{array}{l}\text { Normal physiological } \\
\text { levels/M }\end{array}$ \\
\hline CHOL $^{\text {a }}$ & - & $3.07 \times 10^{-3}$ & $3.10 \times 10^{-3 b}$ to $5.20 \times 10^{-3 c}$ \\
CRH & $8.85 \times 10^{-9}$ & $8.85 \times 10^{-13}$ & $7.70 \times 10^{-13}$ to $2.50 \times 10^{-12 d}$ \\
ACTH & $8.85 \times 10^{-8}$ & $8.38 \times 10^{-8}$ & $2.20 \times 10^{-12}$ to $1.33 \times 10^{-11 e}$ \\
CORT & $2.41 \times 10^{-8}$ & $2.86 \times 10^{-8}$ & $5.52 \times 10^{-8}$ to $6.90 \times 10^{-7 f}$ \\
ALDO & $1.62 \times 10^{-8}$ & $6.07 \times 10^{-10}$ & $4.70 \times 10^{-10}$ to $4.27 \times 10^{-9 g}$ \\
\hline
\end{tabular}

Total blood cholesterol.

${ }^{b}$ http://www.merckmanuals.com/professional/endocrine_and_metabolic_disorders/lipid_disorders/hypolipidemia.html (last accessed September 27, 2014).

${ }^{\mathrm{c}}$ http://www.mayoclinic.com/health/cholesterol-levels/CL00001 (last accessed September 27, 2014).

${ }^{\mathrm{d}}$ Hashimoto et al. (1993).

${ }^{\mathrm{e}} \mathrm{http} / / /$ www.mayomedicallaboratories.com/test-catalog/Clinical+and+Interpretive/8411 (last accessed September 27, 2014). http://www.nlm.nih.gov/medlineplus/ency/article/003695.htm (last accessed September 27, 2014).

${ }_{\mathrm{f}}$ http://www.mayomedicallaboratories.com/test-catalog/Clinical+and+Interpretive/8545 (last accessed September 27, 2014).

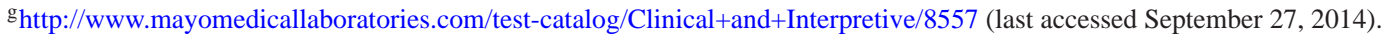

2.2). Results obtained by numerical simulations of HPA axis dynamics under cholesterol regulation are shown in Fig. 5.

As can be seen from the diagrams shown in Fig. 5, the model predicts both quantitative changes in cortisol levels and qualitative dynamic transitions in HPA axis activity under the influence of cholesterol. Starting from normal physiological levels indicated by arrows in Fig. 5, and moving towards higher $k_{1}$ (Fig. 5(a)) or lower $k_{9}$ (Fig. 5(b)), which in either case yields increased cholesterol concentrations, the amplitude of ultradian cortisol oscillations dampens (exemplified here for different values of $k_{1}$, Fig. 5(c-g)). Conversely, the amplitude of cortisol oscillations increases when going in the opposite direction, towards lower $k_{1}$ (Fig. 5(a)) or higher $k_{9}$ (Fig. 5(b)), which corresponds to lower cholesterol levels. In other words, lowering cholesterol levels will increase the amplitude of ultradian oscillations. As long as the ultradian oscillations remain nearly harmonic, occurring with a sufficiently high frequency (one or more ultradian oscillation per hour, as in Figs 4 and 5(c, d)), this will initially enhance the organism's capacity to cope with stress (discussed in detail in Jelić et al., 2008, 2009; Marković et al., 2011a). However, further lowering of cholesterol levels will give rise to high-amplitude relaxation oscillations that occur with rather low frequency (one oscillation in several hours). In this regime, the dynamics of HPA axis 'switches' periodically between two steady states, 'staying' close to one stable steady state for extended times during the day, and occasionally 'jumping' to the other state (as in Fig. 5(f, g)). Hence, the dynamic regulation of HPA axis activity is being diminished. Such dynamic transitions are difficult to detect experimentally/clinically, but may occur in early stages of a disease, recognized as hyper responsiveness of the HPA axis to a challenge.

Here, we note that the diagrams of dynamic transitions shown in Fig. 5 reflect changes at a particular time-point during the day, in this instance at noon ( $t=12: 00$ o'clock). If any other time-point had been considered, these diagrams would have been somewhat different, reflecting differences in the actual concentration of HPA axis hormones, which are different at other times of the day. However, these differences would only affect quantitative values and not critically alter the interpretation of the data, enabling us to draw the same general conclusions. Thus irrespective of the actual time-point that is being 

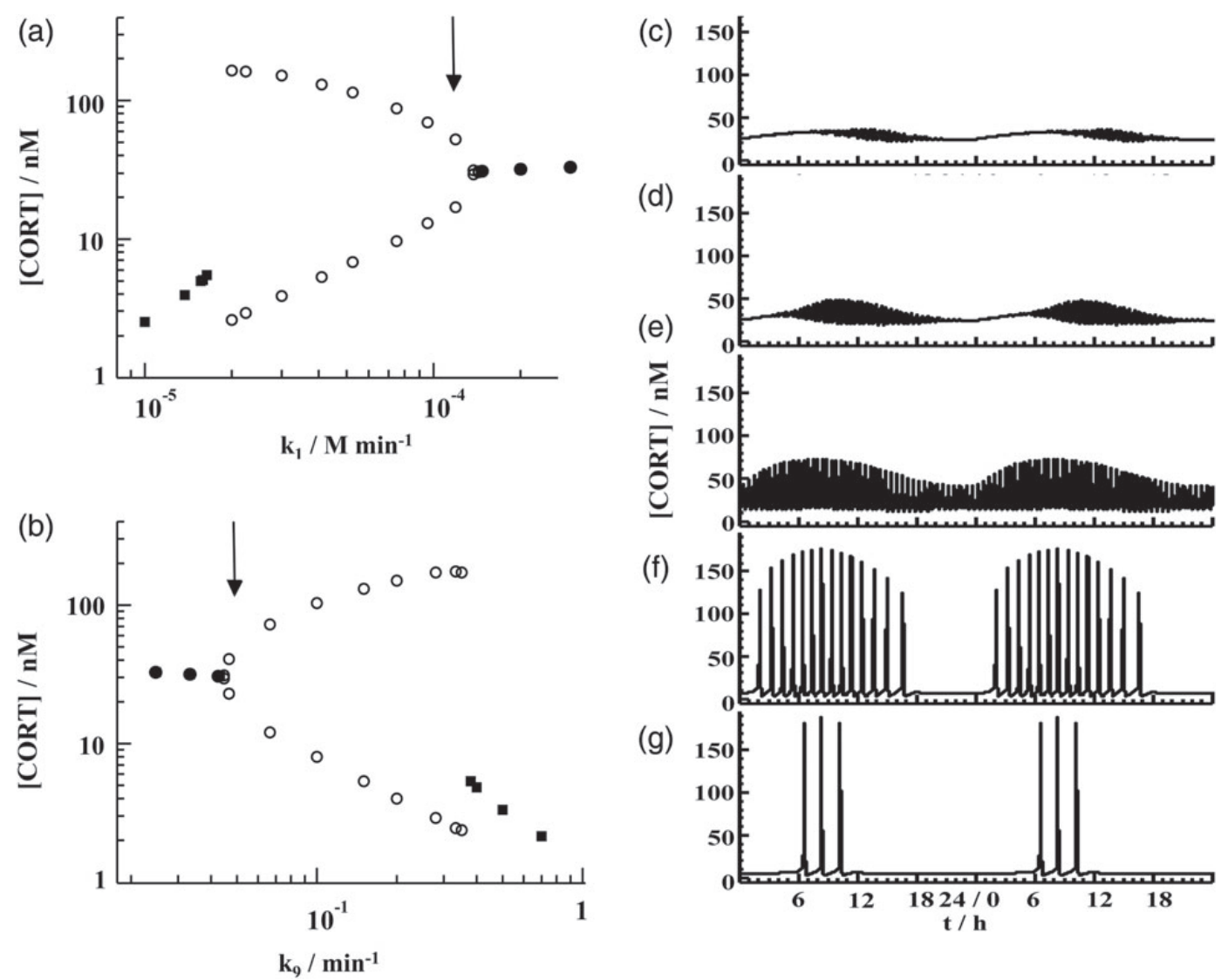

FIG. 5. Changes in HPA axis dynamics under cholesterol regulation. (a) HPA axis dynamics as a function of cholesterol inflow at a constant outflow, $k_{9}=4.5 \times 10^{-2} \mathrm{~min}^{-1}$. (b) HPA axis dynamics as a function of cholesterol outflow at a constant inflow, $k_{1}=1.38 \times 10^{-4} \mathrm{M} \mathrm{min}^{-1}$. For low values of cholesterol inflow/high values of cholesterol outflow, mean daily cholesterol levels are low, the ultradian regulation of HPA axis is being lost and states with low mean daily cortisol levels are being established (full squares). Similarly, the dynamic ultradian regulation is also lost at the high end of cholesterol concentrations, arising due to high-cholesterol inflow/low outflow, where states with high mean daily cortisol levels are being observed (dots). Open circles denote the minimum/maximum of ultradian oscillations at a selected time-point (noon, $t=12: 00 \mathrm{o}$ 'clock). For this particular time-point, the oscillatory ultradian regulation of HPA axis activity is obtained in an interval of mean cholesterol concentrations $3.60 \times 10^{-4} \mathrm{M}<[\mathrm{CHOL}]_{\text {mean }}<3.20 \times 10^{-3} \mathrm{M}$. Arrows indicate oscillatory states of cortisol concentration realized for rate constants values given in Table 1. Daily variation in cortisol concentration for different values of cholesterol inflow: (c) $k_{1}=1.35 \times 10^{-4} \mathrm{M} \mathrm{min}^{-1}$, (d) $k_{1}=1.30 \times 10^{-4} \mathrm{M} \mathrm{min}^{-1}$, (e) $k_{1}=1.00 \times 10^{-4} \mathrm{M} \mathrm{min}^{-1}$, (f) $k_{1}=2.40 \times 10^{-5} \mathrm{M} \mathrm{min}^{-1}$ and (g) $k_{1}=1.68 \times 10^{-5} \mathrm{M} \mathrm{min}^{-1}$.

considered, the diagrams presented in Fig. 5 clearly show that cholesterol effects on HPA axis activity are complex, dependent on which part of the parameter space is being analysed and what property of the system is being considered. They also show why it is difficult to compare experimental results between groups, as they are seldom performed under exactly identical conditions, and why seemingly contradictory conclusions may be reached in different studies.

For example, Auvinen et al. (2012) show that the amplitude of plasma corticosterone (the primary glucocorticoid in rodents) is larger in mice fed on a low-fat diet, than in mice fed on a high-fat 
diet (Fig. 2 in Auvinen et al., 2012), leading to the interpretation that HPA activity is dampened in response to elevated cholesterol levels. Similarly, Tyrka et al. (2012) show that cortisol response to neuroendocrine challenge is reduced in humans with several metabolic risk factors including elevated cholesterol levels (Fig. 3 in Tyrka et al., 2012). Both observations are in agreement with our model predictions, showing that the cortisol oscillation amplitude is smaller for higher cholesterol levels, which are realized for high $k_{1}$ or low $k_{9}$ (Fig. 5). In contrast, a positive correlation was observed between total cholesterol and corticosterone levels (Fig. 3 in Evans et al., 2013), leading to a seemingly opposite conclusion that HPA axis activity is enhanced in response to cholesterol elevation in obesity. On the global level, our model predicts that cortisol concentration increases for increasing cholesterol levels (Fig. 5), paralleling the experimental findings by Evans et al. (2013) showing that mean corticosterone levels increase with cholesterol. At the same time, the amplitude of ultradian oscillations decreases, as shown by Auvinen et al. (2012) and Tyrka et al. (2012). Thus, mathematical modelling suggests that there are no contradictions at the level of primary data, but that seemingly opposite conclusions may be drawn because HPA axis dynamics was analysed in different parts of the parameter space.

Dynamic transitions in HPA axis activity observed for high $k_{1} /$ low $k_{9}$ values (Fig. 5), which reflect conditions where cholesterol levels are high, are associated with the transition of ultradian oscillations through a supercritical Andronov-Hopf bifurcation. The Andronov-Hopf bifurcation was confirmed using the model without circadian rhythms, where the steady states were identified using (14-18) and their stability was determined from the instability conditions (19) and (20). In addition, the AndronovHopf bifurcation was confirmed by numerical simulations, that is from the linear relationship of the amplitude of small limit cycle oscillations squared as a function of the control parameter $\left(\mathrm{A}^{2}=f\left(k_{1}\right)\right.$ and $\mathrm{A}^{2}=f\left(k_{9}\right)$, data not shown).

Properties of the supercritical Andronov-Hopf bifurcation can be linked to some empirically well-established properties of the HPA axis activity. For example, the reversibility of a supercritical Andronov-Hopf bifurcation is consistent with numerous experimental and clinical studies showing that symptoms caused by high-cholesterol levels, which eventually lead to the development of metabolic syndrome, coronary and artery diseases, are reversible and the HPA axis can restore its normal dynamics after decreasing cholesterol levels by returning to healthy lifestyle and/or using cholesterol reducing drugs (see, e.g. a recent review by Akbaraly et al., 2010). Furthermore, the gradual reduction in ultradian oscillation amplitude, which is a hallmark of the Andronov-Hopf bifurcation, is in agreement with the common notion that systems under chronic stress exhibit an ever lower 'excitability' threshold. As the amplitude of ultradian oscillations of HPA axis hormones becomes smaller, the same perturbation may elicit a more intense response because the system's end point is further away from the stable manifold than under conditions where the amplitude of the limit cycle is larger.

In the limit of low cholesterol levels (decreasing $k_{1}$ and increasing $k_{9}$, Fig. 5), the model predicts an initially beneficial increase in the amplitude of ultradian cortisol oscillations. As cholesterol levels continue to fall, the model predicts a decrease in dynamic ultradian regulation of HPA activity, in line with experimentally observed decrease in HPA axis activity that is experimentally verified by Auvinen et al. (2012). In the model, dynamic regulation capacity is being lost through an exponential increase in the period of ultradian oscillations (Fig. 5), which eventually leads to an abrupt break in ultradian regulation at very low cholesterol values. Like hypercholesterolemia (Bhatnagar et al., 2008), hypocholesterolemia (Vyroubal et al., 2008) can be associated with adverse conditions, most notably Addison's disease (Betterle \& Morlin, 2011), but also severe acute and chronic stress (Heim et al., 2000; Gunnar \& Vazquez, 2001; Guilliams \& Edwards, 2010), or intracerebral haemorrhages (Lanterna et al., 2013). However, the impact of hypocholesterolemia on dynamic transitions in HPA axis activity is less 
well documented in the literature, yielding a limited experimental basis for model validation under these conditions.

\subsection{Effect of acute cholesterol stress on the HPA axis dynamics}

In addition to intrinsic daily variations, serum cholesterol levels change in response to interactions with the environment, such as feeding, physical and emotional challenge (Cella et al., 1995). To model the effect of physiological fluctuations in cholesterol concentrations on the HPA axis activity, we induced single-pulse changes in cholesterol concentrations (Fig. 6).

(a)

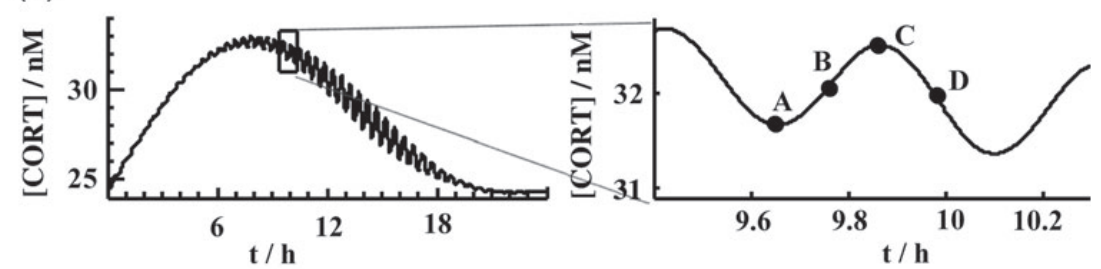

(b)
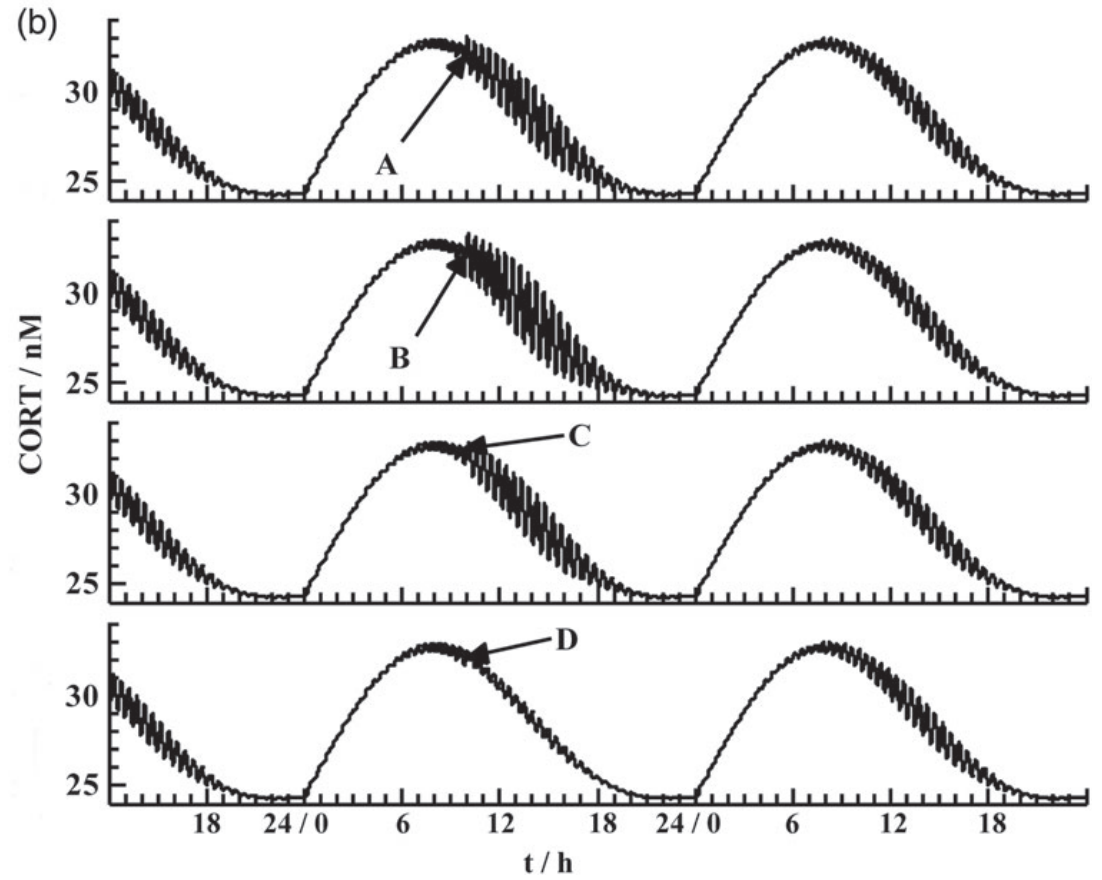

FIG. 6. Response of the HPA system to acute perturbations with cholesterol. (a) Daily variation in cortisol levels (left) and a daytime ultradian cortisol oscillation selected for single-pulse perturbations with cholesterol (right). Points A-D designate the phase of the selected ultradian oscillation at which the cholesterol perturbation was applied. (b) Changes of HPA axis dynamics in response to perturbations with cholesterol inflicted in the: minimum (A), down-to-up inflection point (B), maximum (C) and up-to-down inflection point (D) of the selected ultradian cortisol oscillation shown in (a). The perturbation intensity was the same in all cases, $[\mathrm{CHOL}]=1.875 \times 10^{-4} \mathrm{M}$. 


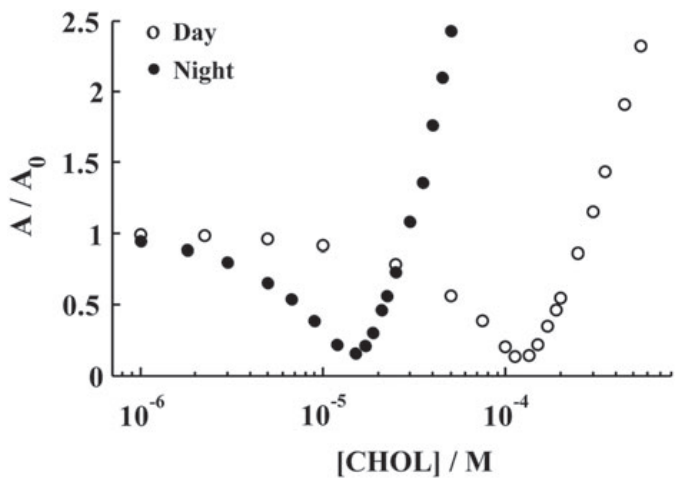

FIG. 7. Differential response of the HPA axis model to cholesterol perturbations applied during the daytime (open circles) and at night (dots). The perturbation intensity was the same in all cases, $[\mathrm{CHOL}]=1.875 \times 10^{-4} \mathrm{M}$. All perturbations were performed at the same circadian phase, at daytime ( $t=10: 00$ o'clock in the morning, open circles) and during the night ( $t=02: 00 \mathrm{o}$ 'clock at night, dots), and at the same ultradian phase, corresponding to the downward inflection point of the selected daytime ultradian oscillation (Point D in Fig. 6(a)). The effect of a perturbation was evaluated by comparing the amplitude $(A)$ of an arbitrarily selected reference oscillation that follows the perturbation with the amplitude $\left(A_{0}\right)$ of the same reference oscillation in an unperturbed system.

As evident from the data presented in Fig. 6, acute changes in cholesterol level affect the global HPA axis activity. The cause-and-effect relationship is complex, since the same change in cholesterol level may induce different effects on HPA axis dynamics - the cortisol amplitude may diminish, increase or remain unaltered, depending on the ultradian phase of the HPA cycle at which the perturbation of cholesterol levels has occurred (Fig. 6). This is a natural feature of systems that exhibit rhythmic behaviour in the vicinity of a supercritical Andronov-Hopf bifurcation, reflecting differences in the actual position of the system on the stable limit cycle with respect to the stable manifold of the saddle focus (Jelić et al., 2008, 2009; Marković et al., 2011a). The empirical observation that the same stimulus does not always introduce the same response of the investigated system is frequently encountered. It is often not recognized and therefore disregarded and treated as an experimental error or attributed as noise.

The model predicts that the effect of cholesterol perturbation on HPA axis activity depends also on the diurnal phase of the HPA cycle (Fig. 7), in agreement with empirical observations that the HPA axis dynamics is more sensitive to perturbations during the rest-phase, i.e. at night, than during the day (Lucassen et al., 2013; Morin et al., 2003). Again, this is a natural consequence of the presence of a supercritical Andronov-Hopf bifurcation and the coupling of ultradian and circadian oscillations. In humans, the amplitude of small limit cycle ultradian oscillations of HPA axis hormones is smaller during the night than during the day (Fig. 4(c)), which means that the 'distance' from the limit cycle to the stable manifold of the saddle focus is 'shorter'. Hence, from a dynamical systems theory point of view, humans are more resilient to perturbations during daylight, which is the active phase of the day, than during the night. Such circadian features of the HPA axis and other systems (e.g. immune system) can be exploited in chronopharmacology (Ohdo, 2010), showing, e.g. that glucocorticoid drugs had more beneficial effects for rheumatoid arthritis patients when administered at night than at the more common time in the morning (Arvidson et al., 1994).

Numerical simulations also show that there is a 'turning' point — for cholesterol concentrations that are smaller than a certain value acute perturbations will lead to reduction in the amplitude of ultradian oscillations of cortisol, whilst larger perturbations will lead to an increase (Fig. 7). Mathematically, this critical value reflects the special 'geometry' of the supercritical Andronov-Hopf bifurcation and 
(a1)

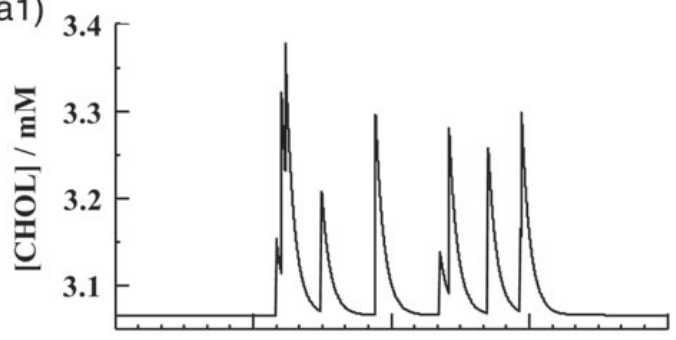

(b1)

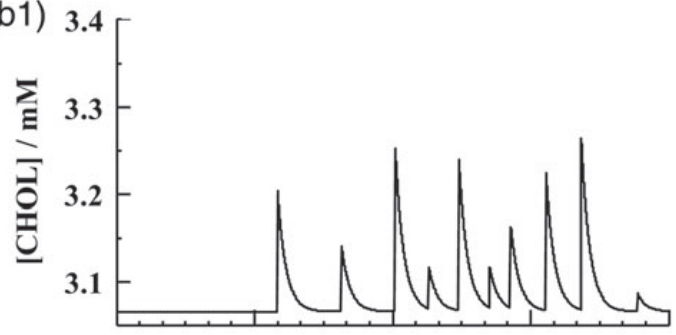

(c1)

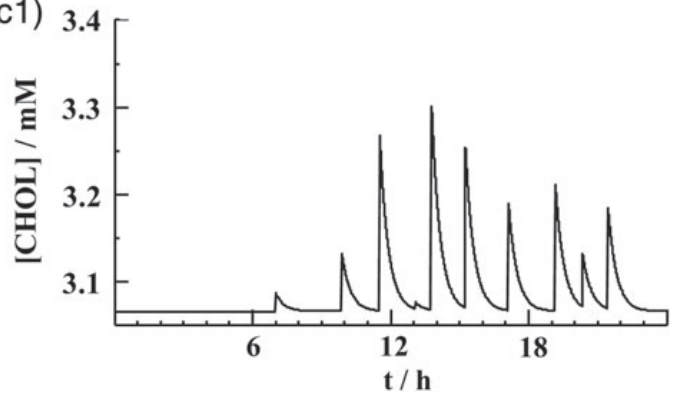

(a2)

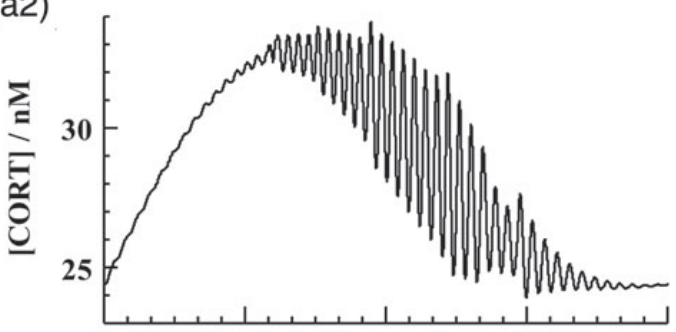

(b2)

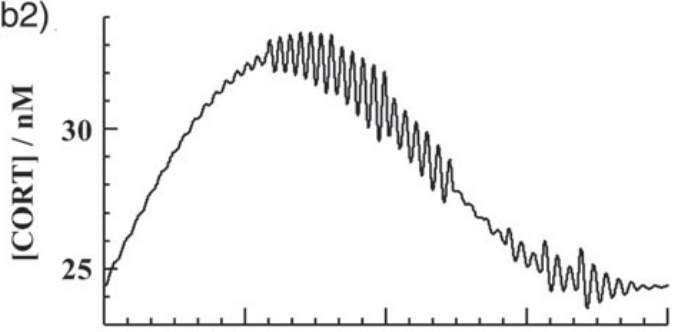

(c2)

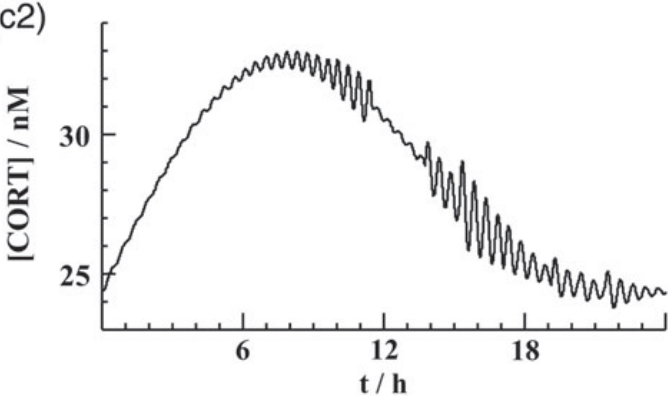

FIG. 8. Response of the HPA system to stochastic acute perturbations with cholesterol. (a)-(c) Time series showing the effect of stochastic perturbations of cholesterol levels (a1-c1) on the HPA axis activity (a2-c2). The time and intensity of cholesterol perturbations were randomly selected as follows. The first perturbation was applied at 07:00 o'clock in the morning, the intensity of cholesterol perturbation was randomly selected to be $0 \leqslant[\mathrm{CHOL}]_{p} \leqslant 0.25 \times 10^{-3} \mathrm{M}$, and the interval between two consecutive pulses was $0 \leqslant t_{p} \leqslant 3 \mathrm{~h}$.

the 'distance' of the limit cycle from the stable manifold of the saddle node-perturbations that end at or close to this manifold will result in the reduction of the oscillation amplitude. However, as the perturbation intensity is increased, the system will end up in states that are further away from the stable manifold, which eventually will result in an increase of the amplitude of the subsequent ultradian oscillation and the limit cycle will no longer be approached from the 'inside' but rather from the 'outside' (see Fig. 3 in Jelić et al., 2009). Physiologically, this means that there is a 'threshold' inbuilt in the regulatory mechanism, and that perturbations of small intensity will be efficiently dampened, whereas large-intensity perturbations will trigger a cascade of well-orchestrated physiological changes and the activation of the stress-response system, the so-called 'fight-or-flight' response.

Similar effects were also obtained in perturbations of the previous model with other dynamical variables of the system, such as CRH or cortisol (Jelić et al., 2009; Marković et al., 2011a). This indicates that the observed behaviour does not reflect some special property of perturbations with cholesterol, 
but is rather an inherent property of the rhythmic HPA axis activity in the vicinity of the supercritical Andronov-Hopf bifurcation.

\subsection{Effect of repeating acute cholesterol stress on the HPA axis dynamics}

To investigate the effect of repeating perturbations of cholesterol levels on the detailed dynamics of the HPA axis activity, we applied random series of cholesterol pulses (Fig. 8(a1-c1)) during the course of numerical integration of (1a-1e) and examined how these perturbations affect the dynamics of the ultradian HPA axis activity (Fig. 8(a2-c2)).

As can be seen, different random series of cholesterol pulses (Fig. 8(a1-c1)) give rise to very different detailed dynamics of the ultradian HPA axis activity (Fig. 8(a2-c2)). These data show that even though in this model ultradian oscillations of HPA axis hormones arise through a purely deterministic mechanism, stochastic external perturbations may lead to the development of intricate dynamical patterns of HPA axis activity, offering a possibility for an additional source of variations in cortisol concentration profiles in one and the same individual from one day to another.

\section{Conclusion}

The simple, 5D model of cholesterol effects on HPA axis activity proposed here gives a succinct representation of a complex dynamical network that integrates the functions of the nervous and endocrine systems at the level of the whole organism. The model reproduces the diurnal and ultradian variations in cholesterol and key HPA axis hormones levels in the peripheral blood circulation, reaching quantitative agreement for all investigated species except for ACTH. The model also closely mimics the complex, experimentally characterized effect of cholesterol on the HPA axis activity under normal physiology and disease conditions.

Compared with preceding models of HPA axis activity from which it was derived (Jelić et al., 2005, 2008, 2009; Marković et al., 2011a,b), the current model shows an improved quantitative agreement with experimentally measured levels of main HPA axis hormones (Table 2), and enables us to quantitatively characterize the effects of cholesterol on HPA axis activity. At the same time, the new model retains all characteristic features of the previous models of the HPA axis dynamics, showing that CRH, cortisol and cholesterol, although of very different basal levels, perturb the HPA axis dynamics in a similar way. Modelling also replicated empirical observations showing that the HPA axis is more sensitive to perturbations during the rest-phase, when the amplitude of ultradian oscillations is smaller.

In this model, the complex regulation of HPA axis activity arises from the intrinsic nonlinearity of the underlying biochemical interactions and the entanglement of investigated species via feedback mechanisms, rather than from any stochastic or noisy input. Modelling shows that the underlying nonlinearity enables the HPA axis to quickly adjust its dynamics in response to the stochastically changing environmental impact, and promptly restore its balance thereafter. Furthermore, modelling also shows that changes in the dynamic regulation of HPA axis activity lead to reduced adaptive potential, which may be a cause or consequence of disease conditions.

Results presented here compellingly show that mathematical modelling and numerical simulations may be a useful tool for understanding how cholesterol levels impinge on the HPA axis dynamics. This intricate relationship may be difficult to study experimentally with sufficiently high-temporal resolution - high frequency of probes sampling may induce stress in an individual, perturbing the HPA axis dynamics and making it difficult to separate the investigated effects from those arising due to the experimental analysis. In further applications, the presented model may be utilized for understanding of how dynamic regulatory mechanisms that underlie the HPA axis function are compromised in a rising 
number of metabolic and psychiatric disorders associated with the HPA axis dysregulation, including insulin resistance, diabetes type II, metabolic syndrome, eating disorders, posttraumatic stress disorder and major depression. Additionally, the introduced model of the HPA axis may also be a useful tool for designing in silico dynamic drug delivery strategies that are synchronized with the HPA axis rhythmicity of an individual, bringing us closer to personalized pharmacotherapy of patients.

\section{Computational Details}

Numerical simulations were performed using the MATLAB ode15s solver that is based on the Gear algorithm for integration of stiff differential equations (Gear, 1971). Absolute and relative tolerance error values were $3 \times 10^{-20}$ and $1 \times 10^{-14}$, respectively. The model was integrated with stricter tolerances in order to minimize numerical artefacts. However, we have verified that the same dynamical behaviour was observed using relative tolerance values of $1 \times 10^{-9}$ and $3 \times 10^{-6}$. Kinetic rate constants $\left(k_{i}, i=1-13\right)$ used in the numerical simulations are as given in Table 1, unless otherwise specified. Whenever possible, the values of kinetic rate constants were the same as in our previous work (Jelić et al., 2005; Marković et al., 2011b). In reactions involving cholesterol, the kinetic rate constants were selected in such a way that the reaction rates of new and corresponding old reactions were adjusted to be about equal. In this way, dynamical properties of the HPA axis core species were preserved, and remained largely unaltered by cholesterol introduction. At the same time, cholesterol influence on the HPA axis activity could be also analysed. In all simulations involving ultradian and circadian rhythms, parameters $d_{1}$ and $d_{2}$ in the circadian rhythm function $D$ had the following values: $d_{1}=0.885$ and $d_{2}=0.957$. Initial conditions for integration of ODEs in all numerical simulations were: $[\mathrm{CHOL}]_{0}=3.00 \times 10^{-3} \mathrm{~mol} \mathrm{dm}^{-3},[\mathrm{CRH}]_{0}=1.00 \times 10^{-12} \mathrm{~mol} \mathrm{dm}^{-3},[\mathrm{ACTH}]_{0}=$ $8.00 \times 10^{-8} \mathrm{~mol} \mathrm{dm}^{-3},[\mathrm{CORT}]_{0}=4.00 \times 10^{-8} \mathrm{~mol} \mathrm{dm}^{-3}$ and $[\mathrm{ALDO}]_{0}=6.00 \times 10^{-10} \mathrm{~mol} \mathrm{dm}^{-3}$.

The orbit diagrams shown in Fig. 5(a, b) were constructed by simulating the HPA axis dynamics for different values of cholesterol inflow. Dynamic states without ultradian oscillations are indicated by solid dots, whereas open circles denote the minimum/maximum of ultradian oscillations. All values correspond to an arbitrarily selected time-point, in this case noon ( $t=12: 00$ o'clock).

To simulate the effect of acute perturbations by cholesterol (Figs 6-8), numerical integration of ODE (1a-1e) was stopped at a specified time point, and new initial conditions for subsequent integration were defined. For the new initial conditions, cholesterol concentration was changed for an indicated amount, whereas concentrations of all other species retained the same values as before.

\section{Acknowledgements}

Support from the Karolinska Institute Research Funds, the Swedish Research Council, the Knut and Alice Wallenberg Foundation, the Rajko and Maj Dermanović Fund, and the Ministry of Education, Science and Technological Development of the Republic of Serbia, grants 172015 and 45001, is gratefully acknowledged.

\section{REFERENCES}

Akbaraly, T. N., Singh-Manoux, A., Tabak, A. G., Jokela, M., Virtanen, M., Ferrie, J. E., Marmot, M. G., Shipley, M. J. \& Kivimaki, M. (2010) Overall diet history and reversibility of the metabolic syndrome over 5 years: the Whitehall II prospective cohort study. Diabetes Care, 33, 2339-2341.

Andersen, M., Vinther, F. \& Ottesen, J. T. (2013) Mathematical modeling of the hypothalamic-pituitaryadrenal gland (HPA) axis, including hippocampal mechanisms. Math. Biosci., 246, 122-138. 
Arvidson, N. G., Gudbjörnsson, B., Elfman, L., Rydén, A. C., Tötterman, T. H., \& Hällgren, R. (1994) Circadian rhythm of serum interleukin-6 in rheumatoid arthritis. Ann. Rheum. Dis., 53, 521-524.

Auvinen, H. E., Romijn, J. A., Biermasz, N. R., Havekes, L. M., Smit, J. W., Rensen, P. C. \& Pereira, A. M. (2011) Effects of high fat diet on the basal activity of the hypothalamus-pituitary-adrenal axis in mice: a systematic review. Horm. Metab. Res., 43, 899-906.

Auvinen, H. E., Romijn, J. A., Biermasz, N. R., Pijl, H., Havekes, L. M., Smit, J. W., Rensen, P. C. \& Pereira, A. M. (2012) The effects of high fat diet on the basal activity of the hypothalamus-pituitary-adrenal axis in mice. J. Endocrinol., 214, 191-197.

Bairagi, N., Chatterjee, S. \& Chattopadhyay, J. (2008) Variability in the secretion of corticotropin-releasing hormone, adrenocorticotropic hormone and cortisol and understandability of the hypothalamic-pituitaryadrenal axis dynamics - a mathematical study based on clinical evidence. Math. Med. Biol., 25, 37-63.

Betterle, C. \& Morlin, L. (2011) Autoimmune Addison's disease. Endocr. Dev., 20, 161-172.

Bhatnagar, D., Soran, H. \& Durrington, P. N. (2008) Hypercholesterolaemia and its management. Br. Med. J., 337, a993.

Breen, M. S., Breen, M., Terasaki, N., Yamazaki, M. \& Conolly, R. B. (2010) Computational model of steroidogenesis in human $\mathrm{H} 295 \mathrm{R}$ cells to predict biochemical response to endocrine-active chemicals: model development for metyrapone. Environ. Health Perspect., 118, 265-272.

Breen, M., Breen, M. S., Terasaki, N., Yamazaki, M., Lloyd, A. L. \& Conolly R. B. (2011) Mechanistic computational model of steroidogenesis in H295R cells: role of oxysterols and cell proliferation to improve predictability of biochemical response to endocrine active chemical-metyrapone. Toxicol. Sci., 123, 80-93.

Cella, L. K., Van Cauter, E. \& Schoeller, D. A. (1995) Effect of meal timing on diurnal rhythm of human cholesterol synthesis. Am. J. Physiol., 269, E878-E883.

Cirelli, C., Gutierrez, C. M. \& Tononi, G. (2004) Extensive and divergent effects of sleep and wakefulness on brain gene expression. Neuron, 41, 35-43.

Clarke, B. L. (1980) Stability of complex reaction networks. Advances in Chemical Physics, vol. 43 (I. Prigogine \& S. A. Rice eds). New York: John Wiley \& Sons, Inc., pp. 1-216.

Clarke, B. L. (1988) Stoichiometric network analysis. Cell Biophys., 12, 237-253.

Clarke, B. L. \& JiAng, J. (1993) Method for deriving Hopf and saddle-node bifurcation hypersurfaces and application to a model of the Belousov-Zhabotinskii system. J. Chem. Phys., 99, 4464-4478.

Conrad, M., Hubold, C., Fischer, B. \& Peters A. (2009) Modeling the hypothalamus-pituitary-adrenal system: homeostasis by interacting positive and negative feedback. J. Biol. Phys., 35, 149-162.

Čupić, Ž., Marković, V., Ivanović, A. \& Kolar-Anić, LJ. (2011) Modeling of the complex nonlinear processes: Determination of the instability region by the stoichiometric network analysis. Mathematical Modelling (C. R. Brennan ed.). New York: Nova Science Publishers, Inc., pp. 111-178.

Debose-Boyd, R. A. (2008) Feedback regulation of cholesterol synthesis: sterol-accelerated ubiquitination and degradation of HMG CoA reductase. Cell Res., 18, 609-621.

Dempsher, D. P., GanN, D. S. \& Phair, R. D. (1984) A mechanistic model of ACTH-stimulated cortisol secretion. Am. J. Physiol., 246, R587-R596.

Evans, J. F., Islam, S., Urade, Y., Eguchi, N. \& Ragolia, L. (2013) The lipocalin-type prostaglandin D2 synthase knockout mouse model of insulin resistance and obesity demonstrates early hypothalamic-pituitary-adrenal axis hyperactivity. J. Endocrinol., 216, 169-180.

Gallo-Payet, N. \& Payet, M. D. (2003) Mechanism of action of ACTH: beyond cAMP. Microsc. Res. Tech., 61, $275-287$.

García-Prieto, M. D., Tébar, F. J., Nicolás, F., Larqué, E., Zamora. S. \& Garaulet, M. (2007) Cortisol secretary pattern and glucocorticoid feedback sensitivity in women from a Mediterranean area: relationship with anthropometric characteristics, dietary intake and plasma fatty acid profile. Clin. Endocrinol. (Oxf.), 66, $185-191$.

Gavrila, A., Peng, C. K., Chan, J. L., Mietus, J. E., Goldberger, A. L. \& Mantzoros, C. S. (2003) Diurnal and ultradian dynamics of serum adiponectin in healthy men: comparison with leptin, circulating soluble leptin receptor, and cortisol patterns. J. Clin. Endocrinol. Metab., 88, 2838-2843. 
GEAR, C. W. (1971) Numerical Initial Value Problems in Ordinary Differential Equations. Englewood Cliffs, NJ: Prentice-Hall, Inc.

Guilliams, T. G. \& Edwards L. (2010) Chronic stress and the HPA axis: clinical assessment and therapeutic considerations. Standard, 9, 1-12.

Gunnar, M. R. \& VAzQuez, D. M. (2001) Low cortisol and a flattening of expected daytime rhythm: potential indices of risk in human development. Dev. Psychopathol., 13, 515-538.

Gupta, S., Aslakson, E., Gurbaxani, B. M. \& Vernon, S. D. (2007) Inclusion of the glucocorticoid receptor in a hypothalamic pituitary adrenal axis model reveals bistability. Theor. Biol. Med. Model., 4, 8.

Hartmann, A., Veldhuis, J. D., Deuschle, M., Standhardt, H. \& Heuser, I. (1997) Twenty-four hour cortisol release profiles in patients with Alzheimer's and Parkinson's disease compared to normal controls: ultradian secretory pulsatility and diurnal variation. Neurobiol. Aging, 18, 285-289.

Hashimoto, K., Nishioka, T., Numata, Y., Ogasa, T., Kageyama, J. \& Suemaru, S. (1993) Plasma levels of corticotropin-releasing hormone in hypothalamic-pituitary-adrenal disorders and chronic renal failure. Acta Endocrinol. (Copenh.), 128, 503-507.

Heim, C., Ehlert, U. \& Hellhammer, D. H. (2000) The potential role of hypocortisolism in the pathophysiology of stress-related bodily disorders. Psychoneuroendocrinology, 25, 1-35.

Hu, J., Zhang, Z., Shen, WJ. \& Azhar, S. (2010) Cellular cholesterol delivery, intracellular processing and utilization for biosynthesis of steroid hormones, Nutr. Metab. (Lond), 7, 47.

Ingalls, B. P. (2013) Mathematical Modelling in Systems Biology: An Introduction, 1st edn. Cambridge, MA, USA: The MIT Press.

Izhikevich, E. M. (2000) Neural excitability, spiking and bursting. Int. J. Bifurcation Chaos, 10, 1171-1266.

Jelić, S., Čupić, Ž. \& KolaR-AnIĆ, LJ. (2005) Mathematical modelling of the hypothalamic-pituitary-adrenal system activity. Math. Biosci., 197, 173-187.

Jelić, S., Čupić, Ž. \& KolaR-Anić, LJ. (2008) Modelling of the hypothalamic-pituitary-adrenal system activity based on the stoichiometric analysis. New Research on Neurosecretory Systems (E. Romano \& S. De Luca eds). New York: Nova Science Publishers, Inc., pp. 225-245.

Jelić, S., Čupić, Ž, Kolar-Anić, LJ. \& Vukojević, V. (2009) Predictive modeling of the hypothalamic-pituitaryadrenal (HPA) function. Dynamic system theory approach by stochiometric network analysis and quenching of small amplitude oscillations. Int. J. Nonlinear Sci. Num., 10, 1451-1472.

Jones, P. J. \& SCHOELler, D. A. (1990) Evidence for diurnal periodicity in human cholesterol synthesis. J. Lipid Res., 31, 667-673.

Keenan, D. M., Licinio, J. \& Veldhuis, J. D. (2001) A feedback-controlled ensemble model of the stressresponsive hypothalamo-pituitary-adrenal axis. Proc. Natl. Acad. Sci. USA, 98, 4028-4033.

Kellendonk, C., Gass, P., Kretz, O., Schutz, G. \& Tronche, F. (2002) Corticosteroid receptors in the brain: gene targeting studies. Brain Res. Bull., 57, 73-83.

Kolar-Anić, Lu., Čupić, Ž., Schmitz, G. \& ANIĆ, S. (2010) Improvement of the stoichiometric network analysis for determination of instability conditions of complex nonlinear reaction systems. Chem. Eng. Sci., $\mathbf{6 5}$, 3718-3728.

Kyrylov, V., Severyanova, L. A., \& Vieirakyrylov, A. (2005) Modeling robust oscillatory behavior of the hypothalamic-pituitary-adrenal axis. IEEE Trans. Biomed. Eng., 52, 1977-1983.

Lanterna, L. A., Spreafico, V., Gritti, P., Prodam, F., Signorelli, A., Biroli, F. \& Aimaretti, G. (2013) Hypocortisolism in noncomatose patients during the acute phase of subarachnoid hemorrhage. J. Stroke Cerebrovasc. Dis., 22, e189-e196.

Lenbury, Y. \& Pornsawad, P. (2005) A delay-differential equation model of the feedback-controlled hypothalamus-pituitary-adrenal axis in humans. Math. Med. Biol., 22, 15-33.

Lightman, S. L. \& Conway-Campbell B. L. (2010) The crucial role of pulsatile activity of the HPA axis for continuous dynamic equilibration. Nat. Rev. Neurosci., 11, 710-718.

Lightman, S. L., Windle, R. J., Ma, X. M., Harbuz, M. S., Shanks, N. M., Julian, M. D., Wood, S. A., Kershaw, Y. M. \& Ingram, C. D. (2002) Hypothalamic-pituitary-adrenal function. Arch. Physiol. Biochem., 110, 90-93. 
LiU, W. M. (1994) Criterion of Hopf bifurcation without using eigenvalues. J. Math. Anal. Appl., 182, 250-256.

Liu, Y. W., Hu, Z. H., Peng, J. H. \& Liu, B. Z. (1999) A dynamical model for the pulsatile secretion of the hypothalamo-pituary-adrenal axis. Math. Comput. Model., 29, 103-110.

Lomax, M. A., Karamanlidis, G., Laws, J., Cremers, S. G., Weinberg, P. D. \& Clarke, L. (2013) Pigs fed saturated fat/cholesterol have a blunted hypothalamic-pituitary-adrenal function, are insulin resistant and have decreased expression of IRS-1, PGC1 $\alpha$ and PPAR $\alpha$. J. Nutr. Biochem., 24, 656-663.

Lu, K., Lee, M. H. \& Patel, S. B. (2001) Dietary cholesterol absorption; more than just bile. Trends Endocrinol. Metab., 12, 314-320.

Lucassen, E. A., Zhao, X., Rother, K. I., Mattingly, M. S., Courville, A. B., De Jonge, L., Csako, G. \& CizzA, G. (2013) Evening chronotype is associated with changes in eating behavior, more sleep apnea, and increased stress hormones in short sleeping obese individuals. PLoS One., 8, e56519.

MAĆEŠIĆ, S., ČUPIĆ, ž. \& KolAR-ANIĆ, LJ. (2012) Model of the nonlinear reaction system with autocatalysis and autoinhibition: stability of dynamic states. Hem. Ind., 66, 637-646.

Makino, S., Налнімото, K. \& Gold, P. W. (2002) Multiple feedback mechanisms activating corticotropin releasing hormone system in the brain during stress. Pharmacol. Biochem. Behav., 73, 147-158.

Marković, V. M., Čupić, Ž., Vukojević, V. \& Kolar-Anić, Lu. (2011a) Predictive modeling of the hypothalamicpituitary-adrenal (HPA) axis response to acute and chronic stress. Endocr. J., 58, 889-904.

Marković, V. M., Čupić, Ž., Vukojević, V. \& Kolar-Anić, LJ. (2011b) The stability of the extended model of hypothalamic-pituitary-adrenal (HPA) axis examined by stoichiometric network analysis (SNA). Russ. J. Phys. Chem. A, 85, 2327-2335.

McAuley, M. T., Kenny, R. A., Kirkwood, T. B. L., Wilkinson D. J., Jones J. J. L. \& Miller, V. M. (2009) A mathematical model of aging-related and cortisol induced hippocampal dysfunction. BMC Neurosci., 10, 26.

Meyer-Hermann, M., Figge, M. T., \& Straub, R. H. (2009) Mathematical modeling of the circadian rhythm of key neuroendocrine-immune system players in rheumatoid arthritis: a systems biology approach. Arthritis. Rheum., 60, 2585-2594.

Miller, W. L. \& Auchus, R. J. (2011) The molecular biology, biochemistry, and physiology of human steroidogenesis and its disorders. Endocr. Rev., 32, 579.

Miller, W. L. \& Chrousos, G. P. (2001) The adrenal cortex. Endocrinology \& Metabolism (P. Felig \& L. Frohman eds). New York: McGraw-Hill, pp. 387-524.

Moreno-Ramos, O. A., Lattig, M. C. \& GonzáLez Barrios, A. F. (2013) Modeling of the hypothalamicpituitary-adrenal axis-mediated interaction between the serotonin regulation pathway and the stress response using a Boolean approximation: a novel study of depression. Theor. Biol. Med. Model., 10, 59.

Morin, C. M., Rodrigue, S. \& Ivers, H. (2003) Role of stress, arousal, and coping skills in primary insomnia. Psychosom. Med., 65, 259-267.

Oнdo, S. (2010) Chronotherapeutic strategy: rhythm monitoring, manipulation and disruption. Adv. Drug Deliv. Rev., 62, 859-875.

Peterson, J. E., Wilcox, A. A., Haley, M. I. \& Keith, R. A. (1960) Hourly variation in total serum cholesterol. Circulation, 22, 247-253.

Postnova, S., Fulcher, R., Braun, H. A. \& Robinson, P. A. (2013) A minimal physiologically based model of the HPA axis under influence of the sleep-wake cycles. Pharmacopsychiatry, 46 (Suppl. 1), S36-S43.

REDINGER, R. N. (2003) Nuclear receptors in cholesterol catabolism: molecular biology of the enterohepatic circulation of bile salts and its role in cholesterol homeostasis. J. Lab. Clin. Med., 142, 7-20.

SAVIĆ, D. \& Jelić, S. (2005) A mathematical model of the hypothalamo-pituitary-adrenocortical system and its stability analysis. Chaos Soliton. Fract., 26, 427-436.

Scheff, J. D., Calvano, S. E., Lowry, S. F. \& Androulakis, I. P. (2012) Transcriptional implications of ultradian glucocorticoid secretion in homeostasis and in the acute stress response. Physiol. Genomics, 44, 121-129. 
SchmitZ, G., Kolar-Anić, LJ. Z., Anić, S. R. \& Čupić, Ž. D. (2008) Stoichiometric network analysis and associated dimensionless kinetic equations. Application to a model of the Bray-Liebhafsky reaction. J. Phys. Chem. A, 112, 13452-13457.

Schulkin, J., Gold, P. W. \& Mcewen, B. S. (1998) Induction of corticotrophin-releasing hormone gene expression by glucocorticoids: implication for understanding the states of fear and anxiety and allostatic load. Psychoneuroendocrinology, 23, 219-243.

Sмith, S. M. \& VALE, W. W. (2006) The role of the hypothalamic-pituitary-adrenal axis in neuroendocrine responses to stress. Dialogues Clin. Neurosci., 8, 383-395.

Sriram, K., Rodriguez-Fernandez, M. \& Doyle, F. J. III (2012) Modeling cortisol dynamics in the neuroendocrine axis distinguishes normal, depression, and post-traumatic stress disorder (PTSD) in humans. PLoS Comput. Biol., 8, e1002379.

Strogatz, S. H. (1994) Nonlinear Dynamics and Chaos: With Applications to Physics, Biology, Chemistry and Engineering. Reading, MA: Perseus Books, pp. 262-263.

Tsigos, C. \& Chrousos, G. P. (2002) Hypothalamic-pituitary-adrenal axis, neuroendocrine factors and stress. J. Psychosom. Res., 53, 865-871.

Tyrka, A. R., Walters, O. C., Price, L. H., Anderson, G. M. \& Carpenter, L. L. (2012) Altered response to neuroendocrine challenge linked to indices of the metabolic syndrome in healthy adults. Horm. Metab. Res., 44, 543-549.

Vinther, F., Andersen, M. \& Ottesen, J. T. (2011) The minimal model of the hypothalamic-pituitary-adrenal axis. J. Math. Biol., 63, 663-690.

Vyroubal, P., Chiarla, C., Giovannini, I., Hyspler, R., Ticha, A., Hrnciarikova, D. \& Zadak, Z. (2008) Hypocholesterolemia in clinically serious conditions-review. Biomed. Pap. Med. Fac. Univ. Palacky Olomouc Czech Repub., 152, 181-189.

Walker, J. J., Terry, J. R. \& Lightman, S. L. (2010) Origin of ultradian pulsatility in the hypothalamic-pituitaryadrenal axis. Proc. R. Soc. B, 277, 1627-1633.

Zarzer, C. A., Puchinger, M. G., Köhler, G. \& Kügler, P. (2013) Differentiation between genomic and nongenomic feedback controls yields an HPA axis model featuring hypercortisolism as an irreversible bistable switch. Theor. Biol. Med. Model., 10, 65.

\section{Appendix}

For the purpose of further analysis, ODE (1a-1e) (main text) are rewritten in the following form:

$$
\begin{aligned}
& \frac{\mathrm{d}[\mathrm{CHOL}]}{\mathrm{d} t}=v_{1}-\left(v_{5}+v_{6}\right)-v_{9} \\
& \frac{\mathrm{d}[\mathrm{CRH}]}{\mathrm{d} t}=v_{2}-\left(v_{4}+v_{10}\right) \\
& \frac{\mathrm{d}[\mathrm{ACTH}]}{\mathrm{d} t}=v_{4}-\left(v_{5}+v_{6}\right)-v_{7}-v_{11} \\
& \frac{\mathrm{d}[\mathrm{CORT}]}{\mathrm{d} t}=v_{5}+v_{7}-v_{8}-v_{12} \\
& \frac{\mathrm{d}[\mathrm{ALDO}]}{\mathrm{d} t}=v_{3}+v_{6}-v_{8}-v_{13}
\end{aligned}
$$

where $v_{m}$ are reaction rates of reaction steps $\mathrm{R}_{m}(m=1-13)$ displayed in Table 1 (main text), that can be expressed as functions of the related rate constants $k_{m}$ and the concentrations of species taking part 
in particular reactions, respectively: $v_{1}=k_{1}, v_{2}=k_{2}, v_{3}=k_{3}, v_{4}=k_{4}[\mathrm{CRH}], v_{5}=k_{5}$ [CHOL][ACTH], $v_{6}=k_{6}[\mathrm{CHOL}][\mathrm{ACTH}], v_{7}=k_{7}[\mathrm{ACTH}][\mathrm{CORT}]^{2}, v_{8}=k_{8}[\mathrm{ALDO}][\mathrm{CORT}]^{2}, v_{9}=k_{9}[\mathrm{CHOL}], v_{10}=$ $k_{10}[\mathrm{CRH}], v_{11}=k_{11}[\mathrm{ACTH}], v_{12}=k_{12}[\mathrm{CORT}]$ and $v_{13}=k_{13}[\mathrm{ALDO}]$.

To derive the instability criteria by SNA, the temporal evolution of the investigated system described by ODE (A.1a-A.1e) is more suitably represented in the matrix form:

$$
\frac{\mathrm{d} x}{\mathrm{~d} t}=\mathbf{J} \boldsymbol{x}
$$

where $\mathbf{x}$ is the concentration vector whose elements $\left[\mathrm{X}_{n}\right]$ are concentrations of $n=1,2, \ldots, 5$ intermediary chemical species of the model (cholesterol (CHOL), CRH, ACTH, cortisol (CORT) and aldosterone $(\mathrm{ALDO})$ ), respectively: $\left[\mathrm{X}_{1}\right]=[\mathrm{CHOL}],\left[\mathrm{X}_{2}\right]=[\mathrm{CRH}],\left[\mathrm{X}_{3}\right]=[\mathrm{ACTH}],\left[\mathrm{X}_{4}\right]=[\mathrm{CORT}]$, $\left[\mathrm{X}_{5}\right]=[\mathrm{ALDO}] ; \boldsymbol{v}$ is a reaction rate vector consisting of 13 reaction rates $v_{m}$ of reactions $(m=1-13)$. The stoichiometric matrix $\mathbf{S}$ is an operator whose elements are stoichiometric coefficients, $\mathbf{S}_{n, m}$, defined as $\mathrm{S}_{n, m}=s_{n, m}^{\mathrm{D}}-\mathrm{s}_{n, m}^{\mathrm{L}}$ where, $\mathrm{s}_{n, m}^{\mathrm{L}}$ and $\mathrm{s}_{n, m}^{\mathrm{D}}$, represent stoichiometric coefficients of the model's intermediary chemical species cholesterol CHOL, CRH, ACTH, CORT and ALDO on the left (L) and right (D) sides of the chemical equation for $\mathrm{R}_{m}(m=1,2, \ldots, 13)$, respectively (see Table 1 , main text). Thus, the stoichiometric matrix $\mathbf{S}$ is given by

$$
S=\left[\begin{array}{ccccccccccccc}
1 & 0 & 0 & 0 & -1 & -1 & 0 & 0 & -1 & 0 & 0 & 0 & 0 \\
0 & 1 & 0 & -1 & 0 & 0 & 0 & 0 & 0 & -1 & 0 & 0 & 0 \\
0 & 0 & 0 & 1 & -1 & -1 & -1 & 0 & 0 & 0 & -1 & 0 & 0 \\
0 & 0 & 0 & 0 & 1 & 0 & 1 & -1 & 0 & 0 & 0 & -1 & 0 \\
0 & 0 & 1 & 0 & 0 & 1 & 0 & -1 & 0 & 0 & 0 & 0 & -1
\end{array}\right]
$$

The first step in SNA analysis is to detect steady-state reaction pathways in the model (R1R13) that are inherently coupled through stoichiometric constraints. In SNA terminology, these elementary reaction pathways are known as extreme currents $\boldsymbol{E}_{i}$ and represent non-redundant subnetworks of the investigated system. They are identified by solving the expression for the steady-state condition:

$$
\left[\begin{array}{l}
\mathrm{d} x_{1} \\
\mathrm{~d} t \\
\frac{\mathrm{d} x_{2}}{\mathrm{~d} t} \\
\frac{\mathrm{d} x_{3}}{\mathrm{~d} t} \\
\frac{\mathrm{d} x_{4}}{\mathrm{~d} t} \\
\frac{\mathrm{d} x_{5}}{\mathrm{~d} t}
\end{array}\right]=\left[\begin{array}{ccccccccccccc}
1 & 0 & 0 & 0 & -1 & -1 & 0 & 0 & -1 & 0 & 0 & 0 & 0 \\
0 & 1 & 0 & -1 & 0 & 0 & 0 & 0 & 0 & -1 & 0 & 0 & 0 \\
0 & 0 & 0 & 1 & -1 & -1 & -1 & 0 & 0 & 0 & -1 & 0 & 0 \\
0 & 0 & 0 & 0 & 1 & 0 & 1 & -1 & 0 & 0 & 0 & -1 & 0 \\
0 & 0 & 1 & 0 & 0 & 1 & 0 & -1 & 0 & 0 & 0 & 0 & -1
\end{array}\right]\left[\begin{array}{c}
v_{1} \\
v_{2} \\
v_{3} \\
v_{4} \\
v_{5} \\
v_{6} \\
v_{7} \\
v_{9} \\
v_{10} \\
v_{11} \\
v_{12} \\
v_{13}
\end{array}\right]_{s s}=\left[\begin{array}{l}
0 \\
0 \\
0 \\
0 \\
0
\end{array}\right] .
$$


By solving (A.4) for the steady-state condition, the extreme current matrix $\boldsymbol{E}$ is obtained:

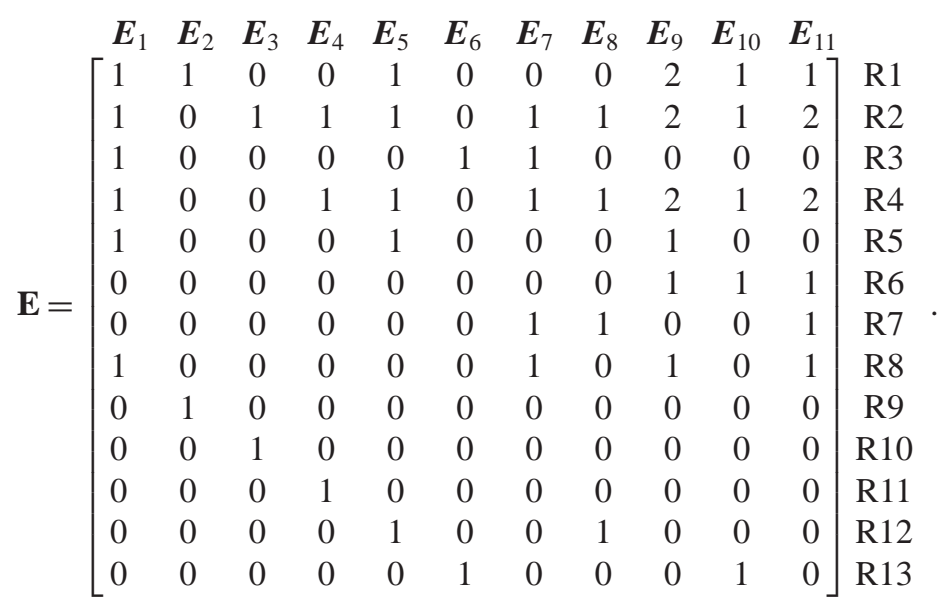

The total number of extreme currents found in this model is 11. Each of the columns $\boldsymbol{E}_{i}(i=$ $1, \ldots, 11)$ actually represents a reaction pathway that can be found in the steady state. For example, $\boldsymbol{E}_{6}$ is the pathway comprising reactions $(\mathrm{R} 3)+(\mathrm{R} 13), \boldsymbol{E}_{9}$ is a reaction pathway of reactions $2(\mathrm{R} 1)+2(\mathrm{R} 2)+2(\mathrm{R} 4)+(\mathrm{R} 5)+(\mathrm{R} 6)+(\mathrm{R} 8)$ and so on.

The contribution of the extreme current $\boldsymbol{E}_{i}$ to a reaction rate at a steady state is designated as a current rate, $j_{i}$. Current rates $j_{i}$ make the components of the corresponding vector $\boldsymbol{j}$. Therefore, using the matrix $\boldsymbol{E}$, reaction rates at the steady state can be expressed as linear combinations of the extreme current rates (Clarke, 1980, 1988; Čupić et al., 2011), in accordance with the equation $\boldsymbol{v}_{\mathrm{ss}}=E j$, where $v_{\mathrm{ss}}$ is a vector of reaction rates at the steady state. Since reaction rates depend on the corresponding rate constants and the concentrations of chemical species, correlations between classic chemical kinetic parameters and the parameters of the SNA is are attained. These correlations can be derived by replacing A.5 in $v_{\mathrm{ss}}=\mathbf{E} \mathbf{j}$, yielding:

$$
\begin{aligned}
& v_{1, \mathrm{ss}}=k_{1}=j_{1}+j_{2}+j_{5}+j_{6}+2 j_{9}+j_{10}+j_{11} \\
& v_{2, \mathrm{ss}}=k_{2}=j_{1}+j_{3}+j_{4}+j_{5}+j_{7}+j_{8}+2 j_{9}+j_{10}+2 j_{11} \\
& v_{3, \mathrm{ss}}=k_{3}=j_{1}+j_{6}+j_{7} \\
& v_{4, \mathrm{ss}}=k_{4}[\mathrm{CRH}]_{\mathrm{ss}}=j_{1}+j_{4}+j_{5}+j_{7}+j_{8}+2 j_{9}+j_{10}+2 j_{11} \\
& v_{5, \mathrm{ss}}=k_{5}[\mathrm{CHOL}]_{\mathrm{ss}}[\mathrm{ACTH}]_{\mathrm{ss}}=j_{1}+j_{5}+j_{9} \\
& v_{6, \mathrm{ss}}=k_{6}[\mathrm{CHOL}]_{\mathrm{ss}}[\mathrm{ACTH}]_{\mathrm{ss}}=j_{9}+j_{10}+j_{11} \\
& v_{7, \mathrm{ss}}=k_{7}[\mathrm{ACTH}]_{\mathrm{ss}}[\mathrm{CORT}]_{\mathrm{ss}}^{2}=j_{7}+j_{8}+j_{11} \\
& v_{8, \mathrm{ss}}=k_{8}[\mathrm{ALDO}]_{\mathrm{ss}}[\mathrm{CORT}]_{\mathrm{ss}}^{2}=j_{1}+j_{7}+j_{9}+j_{11} \\
& v_{9, \mathrm{ss}}=k_{9}[\mathrm{CHOL}]_{\mathrm{ss}}=j_{2}
\end{aligned}
$$




$$
\begin{aligned}
& v_{10, \mathrm{ss}}=k_{10}[\mathrm{CRH}]_{\mathrm{ss}}=j_{3} \\
& v_{11, \mathrm{ss}}=k_{11}[\mathrm{ACTH}]_{\mathrm{ss}}=j_{4} \\
& v_{12, \mathrm{ss}}=k_{12}[\mathrm{CORT}]_{\mathrm{ss}}=j_{5}+j_{8} \\
& v_{13, \mathrm{ss}}=k_{13}[\mathrm{ALDO}]_{\mathrm{ss}}=j_{6}+j_{10} .
\end{aligned}
$$

From (A.6a-A.6m), five independent linear relations given in the main text as $(2 \mathrm{a}-2 \mathrm{e})$ were established. 